\title{
Chronicle of a Soil Bacterium: Paenibacillus polymyxa E681 as a Tiny Guardian of Plant and Human Health
}

\author{
Haeyoung Jeong ${ }^{1,2+}$, Soo-Keun Choi ${ }^{1,2+}$, Choong-Min Ryu ${ }^{1,2 *}$ and Seung-Hwan Park ${ }^{1,2 *}$ \\ 1 Infectious Disease Research Center, KRIBB, Daejeon, South Korea, ${ }^{2}$ Department of Biosystems and Bioengineering, \\ KRIBB School of Biotechnology, Korea University of Science and Technology, Daejeon, South Korea
}

OPEN ACCESS

Edited by:

Camille Eichelberger Granada,

University of Taquari Valley, Brazil

Reviewed by:

Evelise Bach,

Universidade Federal do Rio Grande

do Sul (UFRGS), Brazil

Youn-Sig Kwak,

Gyeongsang National University,

South Korea

Jochen Schmid,

Technische Universität München,

Germany

*Correspondence:

Choong-Min Ryu

cmryu@kribb.re.kr

Seung-Hwan Park

shwan3201@gmail.com;

shpark@kribb.re.kr

${ }^{t}$ These authors have contributed equally to this work

Specialty section:

This article was submitted to

Plant Microbe Interactions,

a section of the journal

Frontiers in Microbiology

Received: 13 October 2018

Accepted: 21 February 2019

Published: 15 March 2019

Citation:

Jeong H, Choi S-K, Ryu C-M and Park S-H (2019) Chronicle of a Soil Bacterium: Paenibacillus polymyxa E681 as a Tiny Guardian of Plant and Human Health.

Front. Microbiol. 10:467. doi: 10.3389/fmicb.2019.00467
The Gram-positive rhizosphere bacterium Paenibacillus polymyxa promotes plant growth and produces various antibiotics. Herein, we review research on this species over the past two and a half decades, and focus on the mechanisms of $P$. polymyxa strain E681, isolated from barley roots in the South Korea in 1995. Strain E681 has outstanding growth-promoting effects on barley, cucumber, pepper, sesame, and Arabidopsis thaliana and produces antimicrobial compounds that protect plants against pathogenic fungi, oomycetes, and bacteria. Induced systemic resistance elicited by treating seeds or roots with strain E681 is a possible mechanism for protecting systemic plant tissues from biotic and other environmental stresses. Genome sequencing has broadened our horizons for antibiotic development and other industrial applications beyond agricultural use. At least six gene clusters for the biosynthesis of antibiotics have been discovered, including polymyxin $(p m x)$, which was recently re-instated as an antibiotic of last resort against Gram-negative drug-resistant bacteria. Three groups of antibiotic synthetases include the gene clusters that encode one for the non-ribosomal peptide polymyxin, fusaricidin, and tridecaptin, another for the lantibiotic paenilan, and the third for a polyketide. We successfully introduced the pmx gene cluster into the surrogate host Bacillus subtilis and created polymyxin derivatives by domain swapping. Furthermore, various E681 derivatives, including a high fusaricidin producer and strains lacking multi-antibiotics production, have been constructed by random mutagenesis and genome engineering. Thus, E681 is an important bacterium that contributes to both plant and human health.

Keywords: PGPR, antimicrobial peptides, induced systemic resistance, polymyxin, non-ribosomal peptide synthetases, polyketides

\section{INTRODUCTION}

A recent paradigm shift upon revisiting the role of soil microbes in plant health suggests that the rhizosphere microbiome is a key determinant of plant health (Berendsen et al., 2012). Plant growth-promoting rhizobacteria (PGPR), which colonize plant roots, draw special attention due to their beneficial effects on crops and ecosystems (Kloepper et al., 2004). Rhizosphere bacteria strains of various genera are designated PGPR. The fluorescent pseudomonads have received special 
interest due to their versatile metabolism, rapid growth, and strong mobility (Bakker et al., 2007). Streptomycetaceae are exceptional antibiotic producers and can suppress plant pathogens. However, the major barriers to agricultural usage of Streptomycetes spp. and Pseudomonas spp. are difficulties associated with mass production in liquid culture, and poor long-term storage due to a short shelf-life, respectively (Emmert and Handelsman, 1999). Bacillus spp. and Paenibacillus spp. are strong candidates for overcoming the drawbacks of Streptomycetes spp. and Pseudomonas spp. (Palaniyandi et al., 2013). Bacillales produce diverse antimicrobial peptides that suppress the growth and fitness of human and plant pathogenic microbes (Ongena and Jacques, 2008; Sumi et al., 2015). Additionally, other metabolites from Bacillales species include human immune activating factors and plant growth regulators (Farag et al., 2013; Levy et al., 2016; Postler and Ghosh, 2017; Sharifi and Ryu, 2018). Among Bacillales species, Paenibacillus spp. have received relatively less attention, since most research has focused on Bacillus species. Due to emerging issues with the re-use and side effects of last resort polymyxin antibiotics, and the occurrence of insects resistant to Bacillus thuringiensis toxins, we recently turned our attention to other Bacillales species.

Species belonging to the Paenibacillus genus were previously re-classified under the genus Bacillus, based on morphological characteristics. However, 16S rRNA sequence analysis (Ash et al., 1991) and PCR probe tests suggested that a group of eleven species should be considered a new genus, Paenibacillus, of which P. polymyxa is the type strain (Ash et al., 1993). In addition, $P$. polymyxa (formerly known as Bacillus polymyxa), is famous for its production of antimicrobial lipopeptides polymyxins, which were described as early as the 1940s and demonstrated to have very strong growth inhibitory activity against Gram-negative bacteria (Storm et al., 1977). In nature, the habitat of Paenibacillus spp. is mainly soil. Due to their multiple beneficial effects, Paenibacillus spp have implications for agriculture, environmental remediation, and even human and animal health. A recent review (Grady et al., 2016) presented a comprehensive summary on research history, scientific impacts, possible applications, and future prospects of Paenibacillus. The description of the genus Paenibacillus was recently amended following the isolation of P. tumbae sp. nov. (Huang et al., 2017). There are 230 approved species in the genus Paenibacillus according to the List of Prokaryotic Names with Standing in Nomenclature $\left(\mathrm{LPSN}^{1}\right)$, and 342 genome sequences are available from the RefSeq database, including 133 genomes without species names as of 17 October 2018.

Out Paenibacillus spp., the type strain P. polymyxa, is regarded as a potential PGPR with a broad host range (Timmusk et al., 2005). One major mechanism of the plant health protection activity of certain $P$. polymyxa strains is the control of plant pathogenic fungi, oomycetes and bacteria by the production of antibiotic compounds (Raza et al., 2008) such as non-ribosomally synthesized lipopeptides polymyxins and fusaricidins. Beyond plant protection, lipopeptides from Paenibacillus spp. have great potential for the treatment of multidrug-resistant (MDR) and

${ }^{1}$ http://www.bacterio.net/index.html, last update on 23 May 2018 human pathogenic bacterial infections (Cochrane and Vederas, 2016). The expansion of publicly available bacterial genome sequences and the development of various genome mining methods (Ziemert et al., 2016) have enabled a systematic approach for the identification of novel and uncharacterized lipopeptides and polyketides from genome sequences (Aleti et al., 2015) and expanded the classical comparative genomic analyses of early available genome sequences, which were restricted to only four P. polymyxa strains E681, CR1, M1, and SC2 (Eastman et al., 2014).

This review presents and discusses current knowledge of the $P$. polymyxa strain E681, a representative strain belonging to the $P$. polymyxa species, including its strain isolation, plant growthpromoting activities, protectant effects, antibiotic production, and genome analysis. The characteristics of strain E681 are compared with those of other $P$. polymyxa strains. In particular, we focused on the identification of antimicrobial compounds and the development of high-yielding strains derived from genome engineering during the 22 years since strain E681 was first isolated in 1995. To the best of our knowledge, E681 is the most studied microorganism belonging to the genus Paenibacillus, and has been consistently studied by $\mathrm{KRIBB}^{2}$ researchers and collaborators in the Republic of Korea (South Korea). Furthermore, the most characterized strain for biosynthetic gene clusters (BGCs) for secondary metabolites as well as their final products among sequenced Paenibacillus strains. Here, we discuss strain E681 in relation to its interactions with plants, and its genome sequencing and comparative genomics, antimicrobial compound production, and industrial applications. The genome sequences of three new $P$. polymyxa isolates and two type strains of Paenibacillus spp., determined by South Korea group, could help expand the phylogenomic perspective of $P$. polymyxa and related species, including $\mathrm{BGC}$ diversity. Average nucleotide identity (ANI)-based analysis suggests that strain E681 should be classified as a novel species along with other strains that have $P$. polymyxa or other species names.

\section{ISOLATION AND CHARACTERIZATION OF Paenibacillus polymyxa STRAIN E681}

As mentioned above, $P$. polymyxa strains have been mostly isolated from soil and rhizosphere, the presumed natural habitats of $P$. polymyxa. For instance, the model strain $P$. polymyxa E681 was isolated from roots of winter barley in South Korea (Ryu and Park, 1997; Ryu et al., 2005b). Among 3179 endospore-forming bacteria isolated after heat treatment at $80^{\circ} \mathrm{C}$ for $1 \mathrm{~h}, 31$ isolates antagonistic against oomycetes pathogens, particularly against Pythium spp. and Phytophthora spp. were obtained. Strain E681 was eventually selected based on its aggressive root colonization capacity compared with other Bacillales species (Choi et al., 2004), and its strong antagonism against a broad spectrum of fungi, oomycetes, and bacteria in vitro. As shown in Figure 1, our previous studies demonstrate that strain E681

\footnotetext{
${ }^{2}$ www.kribb.re.kr
} 

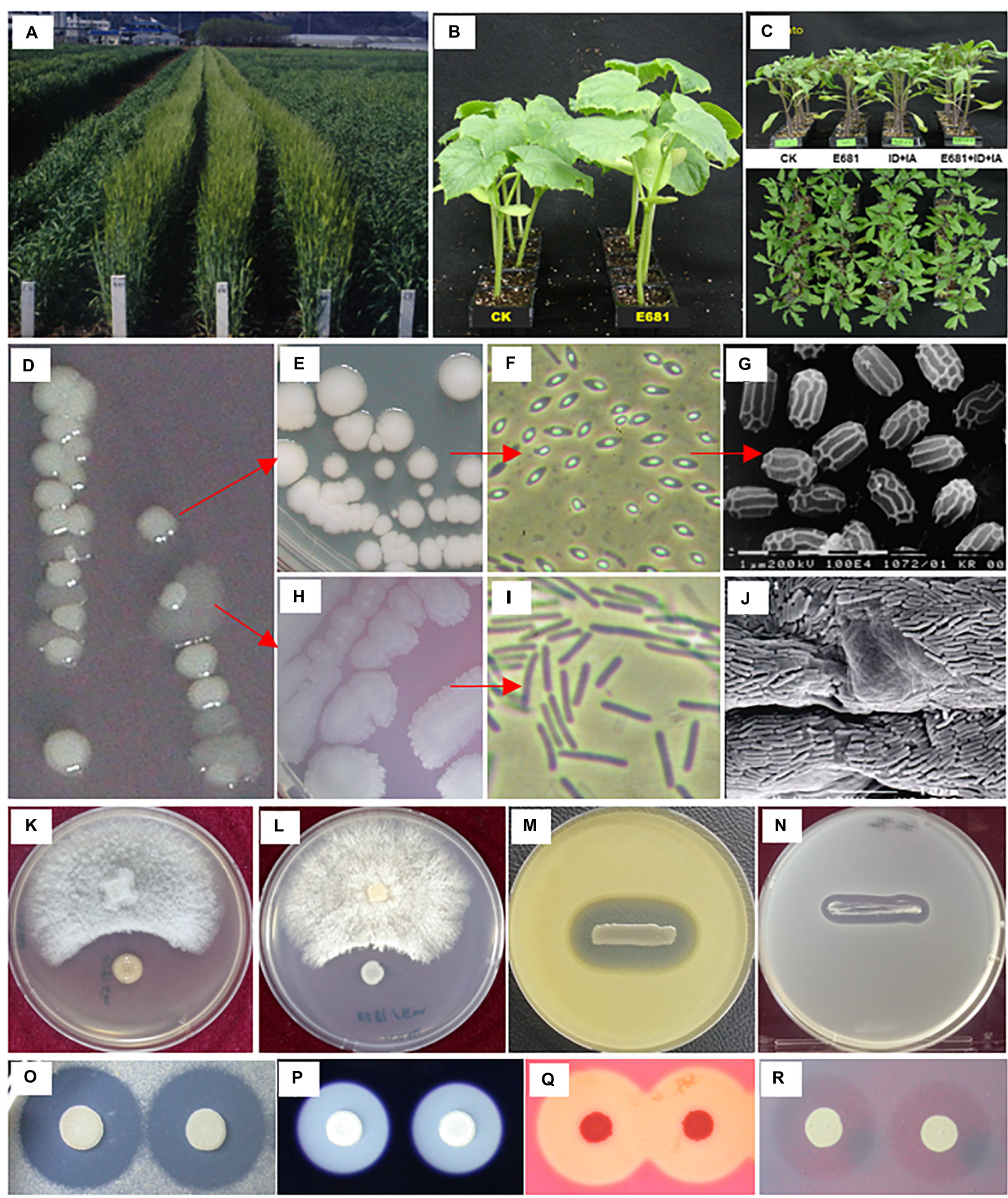

FIGURE 1 | Characteristics of Paenibacillus polymyxa E681 and its plant-beneficial traits. Growth promotion of barley (Cho et al., 1999), cucumber, and tomato by treatment with E681 spores (A-C, respectively); natural phenotypic variation of E681 colonies during growth on tryptic soy broth agar (TSA) plates (D); typical colonies of E681 (E); light and electron microscopy observations of E681 spores (F,G, respectively); flat variant colonies of E681 (H) and light microscopy observation of cells of variant colonies (I); scanning electron microscopy of E681 colonies in cucumber roots (J) (Choi et al., 2004); antimicrobial activity of E681 against Fusarium oxysporum (K), Rhizoctonia solani (L), Micrococcus luteus (M), and Escherichia coli (N); extracellular enzyme activities of protease (0), amylase $\mathbf{( P )}$, cellulose (Q), and mannanase (R). Seeds were soaked with $10^{7-9}$ E681 spores and cells used to assess the plant growth-promoting and root-colonizing abilities of E681. To test antifungal activity, mycelial plugs of fungal pathogens and pre-grown E681 cells were spot-inoculated on a potato dextrose agar plate. To test antibacterial activity, pre-grown E681 cells were streaked on TSA pre-inoculated with bacterial cells at a final concentration of $10^{6} \mathrm{cfu} / \mathrm{ml}$. The extracellular enzyme activities were analyzed by spot-inoculating E681 cells onto TSA plates containing substrates. 
promotes plant growth when seeds are soaked in bacterial suspension, displays antagonism against plant pathogenic fungi, oomycetes, and bacteria, produces various extracellular hydrolytic enzymes, and exhibits potent root colonization activity of various crop species under soil conditions (Ryu and Park, 1997; Choi et al., 2004; Cheong et al., 2005; Ryu et al., 2005a,b). The production of extracellular hydrolytic enzymes by strain E681 indicates that it has potential for use in industrial applications and as a biological control agent against plant pathogenic fungi and bacteria. Furthermore, strain E681 secretes diverse hydrolytic enzymes including proteases, amylases, cellulases, and mannanases, which assist bacterial competence in rhizosphere (Bach et al., 2016; Figures 10-R). Additionally, such enzymes are recognized to have important industrial applications (Grady et al., 2016). Because it has great potential in agriculture, biomedicine, and industry, E681 was the first strain to have its genome completely sequenced, under the original label of $P$. polymyxa (Kim et al., 2010).

It is also worth noting that $P$. polymyxa strains often display a diverse colony shape when grown on complex agar media. Typically, colony formation on solid medium results in a variable morphology, referred to as phase variation or phenotype variation without genetic alteration (Figures 1D,E,H). Once the colony shape changes to a flat form, spores cannot be formed under normal culture conditions (Figure 1I) and antimicrobial activity and extracellular enzymatic activity are significantly reduced (data not shown). Initially, colonies with different shapes were suspected to be contaminants, but genome sequencing detected no differences in nucleotide sequence between the genomes of the two colony morphology types, indicating that they were identical (data not shown).

Additionally, the production of volatile compounds is a key factor mediating inter-kingdom interactions, leading to the elicitation of induced systemic resistance (ISR) in plants (see section "Interactions With Plants and Soil Microorganisms"). To understand the genome functions responsible for adaptation to a plant-associated lifestyle, and to prospect for potential metabolites and gene products that can be used for agricultural and biomedical purposes, the complete genome sequence was determined using conventional Sanger sequencing methodology (see section "Genomics of P. polymyxa E681"). Genome sequence information revealed that E681 harbors an array of BGCs for both known and unknown compounds. At least five of these have since been characterized in terms of both the chemical structures of the compounds and BGC organization (see section "Antimicrobial Compound Production").

\section{INTERACTIONS WITH PLANTS AND SOIL MICROORGANISMS}

In this section, we summarize evidence showing that $P$. polymyxa E681 promotes plant growth and protects against plant pathogens, and compare its properties with those of other strains of $P$. polymyxa. The possible mechanisms underpinning plant responses to $P$. polymyxa are also discussed.

\section{Promotion of Plant Growth}

In early studies on P. polymyxa in the mid-1990s, many scientists explored whether $P$. polymyxa promotes plant growth. Seed treatment with $P$. polymyxa on cucumber as a model crop increased shoot fresh weight by $60 \%$ compared with water controls in greenhouse experiments (Ryu and Park, 1997; Ryu et al., 2005b). Furthermore, drenching soil with $10^{8-9}$ colonyforming units $(\mathrm{CFU}) / \mathrm{ml}$, strain E681 increased height, fresh weight, and dry weight by $3-38 \%$ in $3-$, $4-$, and 5 -week-old pepper seedlings under greenhouse and field conditions (Hahm et al., 2012). Field trials on sesame plants were also conducted by soaking seeds with P. polymyxa strain E681, which improved the yield by 2.85-fold vs. water controls (Ryu et al., 2006). Similarly, seed treatment with strain E681 increased seedling growth and grain yield in barley and wheat after overwintering (Ryu and Park, 1997). Besides crop plants, strain E681 enhanced total leaf surface area in the model plant Arabidopsis thaliana in vitro [on Murashige and Skoog (MS) agar medium] (Ryu et al., 2005a). In the same study, in two separate experiments (direct seeding with vernalized seeds, and 4-week-old seedlings), treating soil with E681 improved foliar fresh weight by more than $30 \%$ vs. water controls (Ryu et al., 2005a). Similarly, plant growth promotion was observed when $A$. thaliana seeds were directly seeded in soil amended with $10^{8} \mathrm{CFU} / \mathrm{ml}$ E681; fresh weight reached $218 \mathrm{mg}$ at 3 weeks after seeding, compared with only $50 \mathrm{mg}$ for controls (Ryu et al., 2005a). Later, soil drenching application of strain E681 achieved similar effects on plant growth (Kwon et al., 2016).

The mode of action of PGPR-mediated plant growth promotion, including that mediated by $P$. polymyxa, has been investigated. Two mechanisms have been advanced: (1) Direct plant growth promotion via bacterial secretion of mimic phytohormones and bacterial nitrogen fixation and (2) Indirect plant growth promotion via PGPR suppression of plant pathogens that cause plant disease. Previous studies demonstrated that siderophore production plays an important role in plant growth promotion and biocontrol. However, genome-wide analysis of strain E681 failed to reveal siderophore non-ribosomal peptide synthetase (NRPS) genes (data not shown).

\section{Mimic Phytohormone Secretion}

Previously mimic plant hormones secreted from PGPR were reported (Lugtenberg and Kamilova, 2009). Plant hormones released by $P$. polymyxa strain E681 include auxins such as indole-3-acetic acid (IAA). Auxins and their intermediates including IAA synthesized by PGPR are key determinants of plant growth stimulation. However, in Paenibacillus spp., little is known about the genetic basis of the key enzymes responsible for IAA biosynthesis. Ethyl acetate extraction and high-performance liquid chromatography (HPLC) analysis have revealed that the IAA and tryptophol (TOL) contents of E681 are 11.84 and $2.30 \mu \mathrm{g} / \mathrm{ml}$, respectively (Phi et al., 2008b). Analysis of IAA intermediates revealed the presence of indole-3-pyruvic acid 
(IPA), indole-3-aldehyde (IAAld), indole-3-lactic acid (ILA), and TOL. Comparative genome analysis of strain E681 with other P. polymyxa strains revealed four genes that synthesize IPA, an IAA intermediate. IPA production was demonstrated in Escherichia coli BL21(DE3) heterologously expressing the IPA decarboxylase (ipdC) gene from the E681 genome (Phi et al., 2008a). However, even though strain E681 was shown to secrete IAA into the culture media, whether plants perceive IAA or its intermediates, and whether this resulted in activation of plant hormonal signaling pathways, remained unknown. Recent proteome analysis of $A$. thaliana seedlings treated with strain E681 revealed augmentation of tryptophan-derived IAA biosynthesis pathway-related proteins ASB1, GSTF6, CYP71B15, and NIT1, which catalyze production of indole-3acetaldoxime (IAOx) (Kwon et al., 2016). Furthermore, liquid chromatography-mass spectrometry (LC-MS) confirmed indole3 -acetonitrile (IAN) and IAA production following activation of protein expression in strain E681. Genetic evidence for the modulation of endogenous IAA signaling was also confirmed by the auxin-insensitive Arabidopsis eir1-3 mutant (Ryu et al., 2005a). Taken together, this evidence indicates that auxins released by E681 play an important role in E681-elicited plant growth promotion. Additionally, cytokinins, pyrroloquinoline quinone (PQQ), and long-chain bacterial volatiles are candidate bacterial factors for plant growth promotion by strain E681 (Seul et al., 2007; Lee et al., 2012). Interestingly, bacterial proteome analysis following barley root exudate stimulation of $A B C$ transporters indicated activation of protein secretion in a plant signal-dependent manner (Seul et al., 2007). However, the exact amounts of plant growth regulators, such as plant hormones, present in the rhizosphere following colonization of the plant root system by E681 are yet to be measured in situ directly, and whether the quantities of metabolites in root exudates are sufficient for initiating auxin signaling in plants remains unknown.

\section{Non-symbiotic Nitrogen Fixation}

Free-living nitrogen fixation is a well-known mode of action on the growth promotion of rhizosphere Bacillus spp. and Paenibacillus spp. (Ruschel et al., 1975). Previously, nitrogen fixation by root-colonizing $P$. polymyxa was reported to be a major mechanism for the promotion of the growth of diverse plant species including lodgepole pine, wheat, sugarcane, and canola (Ruschel et al., 1975; Heulin et al., 1994; Timmusk et al., 2005; Bal and Chanway, 2012a,b; Padda et al., 2017). Despite recent advances in our understanding of symbiotic nitrogen fixation, such as rhizobia and mycorrhizae-legume plant symbiosis and non-symbiotic nitrogen fixation by free-living Gramnegative bacteria, Azotobacter spp., and Azospirillum spp., spore-forming Gram-positive bacteria-mediated nitrogen fixation has not been extensively investigated (Steenhoudt and Vanderleyden, 2000; Schmitz et al., 2002; Mus et al., 2018). The gene cluster that confers nitrogen fixation in $P$. polymyxa could provide a good model for understanding the mechanisms of nitrogen fixation by free-living spore-forming Gram-positive bacteria.

\section{Emission of Bacterial Volatile Compounds}

It is also noteworthy that metabolic cross-talking occurs between exopolysaccharide (EPS) and 2,3-butanediol biosynthesis (Okonkwo et al., 2018). Previously, bacterial volatile compounds emitted from strain E681 into the headspace of flasks were shown to enhance seedling growth of $A$. thaliana (Seul et al., 2007; Lee et al., 2012). The major bacterial volatile compounds from strain E681 were 2,3-butanediol and tridecane, a C13 long-chain fatty acid volatile. $P$. polymyxa strain DSM 365 , a known efficient producer of 2(R),3(R)-butanediol, has its genome completely sequenced (Xie et al., 2015). From the industrial point of view, it is noteworthy that $P$. polymyxa, a non-pathogenic 2,3-butanediol producer, is one of the rare organisms that produces the $\mathrm{R}$ form isomer of 2,3-butanediol in pure form without the need for genetic engineering. By contrast, Klebsiella pneumonia, a serious human pathogen, considered a promising organism for the mass production of 2,3-butanediol (Garg and Jain, 1995). However, the pathogenic characteristics of encapsulated K. pneumonia are considered an obstacle to its industrial applications (Jung et al., 2013). 2,3-Butanediol is an important intermediate metabolite that modulates the secretion of bacterial secondary compounds by stabilizing biosynthesis-related enzymes (Garg and Jain, 1995). Recently, metabolic engineering of this strain to disrupt the $s a c B$ gene encoding levansucrase, the enzyme responsible for the biosynthesis of the major EPS of $P$. polymyxa, levan, resulted in up to a $27 \%$ increase in 2,3-butanediol production when it was grown on sucrose (Okonkwo et al., 2018). These data indicate that increased bacterial volatile production can be used to improve PGPR capacity on plant growth promotion. Similar to other bacterial species, $P$. polymyxa EPS plays a critical role in the root colonization of peanut plants and elicits plant defense against crown rot disease (Haggag, 2007). Discovering the antagonistic regulatory mechanisms of EPS production and bacterial volatile 2,3-butanediol production led to modulating $P$. polymyxa-mediated plant growth and health improvements. Rütering et al. (2017) characterized the complete gene cluster $(\sim 34 \mathrm{~kb})$ for the biosynthesis of heteropolysaccharides, another kind of EPS, from DSM 365 (Rütering et al., 2018), which appears to be widely distributed among Paenibacillus species (see section "Requirement for a New Phylogenomic Position for Strain E681 and Other Related Paenibacillus spp. Strains"). Fine-tuning of bacterial physiology though the engineering of bacterial regulatory networks will help maximize the contribution of P. polymyxa to crop productivity.

\section{Suppression of Plant Pathogens}

Spore-forming bacteria isolated from winter barley and wheat were shown to inhibit diverse plant pathogenic fungi, oomycetes, and bacteria, and exhibited promising biological control potential under field conditions (Ryu et al., 2005b). Following isolation of P. polymyxa E681, strong suppression of soil-borne pathogens Pythium ultimum, Rhizoctonia solani, and Fusarium oxysporum was detected in vitro, accompanied by a significant reduction in damping-off symptoms caused by these oomycetes and fungal pathogens (Ryu et al., 2005b). In this study, pelleting sesame seeds 
with strain E681 and pelleting materials such as clay and talc improved seed germination under greenhouse and field conditions. Field trials demonstrated increased health by $92 \%$, compared with $24 \%$ for controls that suffered heavy fungal infection, leading to reduced health and crop yield (Ryu et al., 2006). At the end of the growing season, the sesame yield of plants pelleted with strain E681 was $77 \mathrm{~kg} / 1000 \mathrm{~m}^{2}$, compared with only $24 \mathrm{~kg} / 1000 \mathrm{~m}^{2}$ for the conventional treatment. Thus, strain E681 is a potent biological control agent under both greenhouse and field conditions, and the biocontrol mechanism can be considered to be caused by the production of a variety of antimicrobials discussed in detail below (see section "Antimicrobial Compound Production") and extracellular hydrolytic enzymes production (see section "Isolation and Characterization of Paenibacillus polymyxa Strain E681"; Figure 1).

\section{Augmentation of the Plant Immune System}

Systemic activation of plant innate immunity elicited by PGPR. ISR capacity against diverse pathogens of numerous crop and model plant species has been reported for many PGPR. The minimum criterion for ISR is spatial separation between inoculation of PGPR and the site of pathogen challenge (Kloepper et al., 2004). In 2012, soil drenching with a bacterial suspension of strain E681 protected pepper plants against bacterial spot disease caused by Xanthomonas axonopodis pv. vesicatoria on aboveground parts (foliar parts such as stems and leaves) (Hahm et al., 2012). Due to limited genetic and molecular knowledge on pepper plants, despite the option of gene knock-down techniques such as virus-induced gene silencing, $A$. thaliana was employed as a model plant to perform genome-wide wholeproteome analysis using two-dimensional electrophoresis (2DE) in conjunction with matrix-assisted laser desorption-ionizationtime of flight (MALDI-TOF) MS (Kwon et al., 2016). Overall, root colonization by strain E681 yielded 17 and 24 differentially expressed protein spots in roots and shoots, respectively. Subsequent transcriptome and metabolite production analyses confirmed that strain E681 modulates the expression of PDF1.2 and camalexin proteins involved in jasmonic acid (JA) signaling, and contributes to ISR capacity against necrotrophic pathogens such as Botrytis cinerea (Kwon et al., 2016). Additional abiotic stress-related proteins and antioxidant enzymes including GST, GPX8, APX1, PER43, and CML42 were also induced by treatment with strain E681, indicating enhanced systemic tolerance against environmental stresses including drought, salt, and cold (Yang et al., 2009).

\section{Involvement of Auxin in ISR}

Similar to the mechanism underpinning plant growth promotion, strain E681 elicits ISR via volatile compounds and auxins (Seul et al., 2007; Phi et al., 2008b; Lee et al., 2012; Park et al., 2013). As described above, large amounts of IAA and its intermediate IPA are released from E681 (Seul et al., 2007; Phi et al., 2008b). The IAA-related pathway contributes not only to plant growth, but also to plant defenses via. camalexin biosynthesis (Fu and Wang, 2011). In the A. thaliana root system, strain E681 induces ASB1, GSTG6, and CYP71B15, which are related to the tryptophan-derived camalexin biosynthesis pathway (Kwon et al., 2016).

\section{Production of Bacterial Volatiles, Tridecane and 2,3-Butanediol}

Strain E681 also produces volatile compounds that elicit ISR against Pseudomonas syringae pv. tomato and $P$. syringae $\mathrm{pv}$. maculicola in $A$. thaliana (Lee et al., 2012). Of 30 volatile compounds, $10 \mathrm{mM}$ and $100 \mu \mathrm{M}$ C13 tridecane was found to be largely responsible for priming plant immunity, and pretreatment with $10 \mathrm{mM}$ tridecane and strain E681 volatiles induced PR1 and VSP2 expression earlier and more strongly following pathogen challenge. These phenomena are referred to as "defense priming" and "plant memory" (Crisp et al., 2016). We also demonstrated that E681-derived long hydrocarbon hexadecane triggered an ISR response that was stronger than the response triggered by acetoin or 2,3-butanediol (Park et al., 2013). Furthermore, pre-application of E681 on pepper roots increases plant insect susceptibility against aphid leaf infestation (Kim et al., 2016). More recently, P. polymyxa strain DSM 365 was shown to increase strongly ISR against an oomycetes pathogen Phytophthora parasitica (Park et al., 2018). The major bacterial determinant for inducing ISR was 2,3-butanediol. Direct leaf infiltration with 2,3-butanediol protected tobacco plants against Phytophthora parasitica in the root system through downregulation of reactive oxygen species biosynthesis-related genes and pathogenesis-related (PR) gene expression (Park et al., 2018). As described above (Okonkwo et al., 2018), engineered DSM 365, an 2,3-butanediol producer, can be used to improve ISR against diverse pathogens. Thus, collectively, evidence suggests that strain E681 strongly modulates the plant immune system. Analysis of E681-elicited ISR should be expanded to other crop plants in greenhouse and field trials in the future.

\section{Interactions With Fungi}

In soil and rhizosphere environments, interactions between PGPR and other soil microorganisms are complex and can affect the beneficial effects of the bacterium. In a study on the interactions between $P$. polymyxa E681 and fungi, it was found that Penicillium citrinum could induce swarming motility in P. polymyxa (Park et al., 2008). We found that citrinin, a mycotoxin produced by the fungus, was involved in the induction of $P$. polymyxa motility via transcriptional activation of genes related to the expression of flagella. This finding provides insight into the interaction mechanisms among microorganisms in natural environments.

\section{GENOMICS OF P. polymyxa E681}

\section{Genome Sequencing of P. polymyxa E681}

In the 1990s, our group participated as a member of the international consortium in genome sequencing of the Grampositive model organism Bacillus subtilis (Kunst et al., 1997) using classical clone-based Sanger sequencing methodologies. Despite a positive outcome and compatibility with genomic 
research on the genus Bacillus and related microbes, obtaining complete bacterial genomes remained challenging until the advent of next-generation sequencing technologies that became available in the early 2000s. Complete genome sequencing of P. polymyxa E681 (Kim et al., 2010) was one of the earlier government-funded genome research programs (Jeong et al., 2008), performed in collaboration with GenoTech Corporation (Daejeon, South Korea). Because the first version of the complete genome sequence, which lacked plasmid sequences, obtained in April 2005, was based on only 6.7-fold coverage $(\sim 62,000$ chromatograms) produced using plasmid/fosmid/BAC libraries, the error rate was as high as 10.74 errors/10 kb. Sequencing errors located in low-quality regions were gradually corrected using manual PCR/Sanger sequencing, and finally, in 2009, Illumina GA IIx sequencing (76 bp single-ended sequences, totaling $2.4 \mathrm{~Gb}$ ) was employed to dramatically improve sequence quality. Read mapping and variant detection were conducted using the MAQ package (Li et al., 2008), followed by confirmatory PCR/Sanger sequencing, and the resulting genome sequence was the first to be publically released for strain E681 (GenBank CP000154.1, 5,394,884 bp, September 2010). The sequence was updated in July 2013 (CP000154.2), in which a $\mathrm{T}(17) \rightarrow \mathrm{T}(16)$ correction rectified a false frameshift at spoIIIAF (PPE_02850, now renumbered as PPE_05995). When Illumina reads were re-mapped onto CP000154.2 using the current version of CLC Genomics Workbench (11.0), no variants were found, implying high confidence for the current release of the E681 genome.

Before the public release, preliminary in-house genome annotation was circulated internally to help identify genes responsible for plant interactions or antibiotic production. For the second version (CP000154.2), the NCBI Prokaryotic Genome Annotation Pipeline (PGAP) was employed and genes were edited based on comparison with the previous version. A minor annotation update without genome sequence correction was made in January 2018 to assign gene and product names systematically to those involved in the biosynthesis of antimicrobial secondary metabolites.

\section{Requirement for a New Phylogenomic Position for Strain E681 and Other Related Paenibacillus spp. Strains}

Early taxonomic allocation of E681 to P. polymyxa species was solely based on $16 \mathrm{~S}$ rRNA gene sequence analysis. However, recent species demarcation approaches allow strains sharing genome similarity with the type strain of a particular species above a certain criterion to be assigned that species name. Genome similarity is often expressed as ANI or other measures (Goris et al., 2007; Richter and Rossello-Mora, 2009), and 95 or 96\% pairwise ANI values are commonly adopted as threshold values. Since no other genomes for $P$. polymyxa species were available when working on the draft genome sequence of strain E681, our group determined genome sequences for two other type strains, namely, P. polymyxa ATCC $842^{\mathrm{T}}$ (Jeong et al., 2006, 2011) and $P$. peoriae KCTC $3763^{\mathrm{T}}$ (Jeong et al., 2012), for comparison with that of E681. The latter was chosen because the $16 \mathrm{~S}$ rRNA sequence of E681 is most similar to that of the
P. peoriae type strain (99.46\%). Unexpectedly, the ANI score between E681 and ATCC $842^{\mathrm{T}}$ calculated using JSpecies (Richter and Rossello-Mora, 2009) was only $86.66 \%$, which implied that E681 may belong to another Paenibacillus species. By the end of 2012, two more complete genome sequences of $P$. polymyxa species became available, namely, those of SC2 (Ma et al., 2011) and M1 (Niu et al., 2011). While these two strains were very similar in terms of ANI score $(>99.8 \%)$ and overall genome organization, they were distinct from both E681 and ATCC $842^{\mathrm{T}}$. Specifically, the ANI value between E681 and M1 (and also SC2) was below 90\%. In other words, no genomes with the original label of $P$. polymyxa, available before 2012, could be assigned to the same species as E681. Thus, we had to await the availability of additional genomes to accurately determine the phylogenetic position of E681. Since then, the genomes of P. polymyxa var. colistinus KCCM 40454 (=ATCC 21830; POVT01), P. polymyxa F4 (POVS01), and P. peoriae HS311 (CP011512-3) have been sequenced by our group to expand current knowledge on Paenibacillus strains for plant probiotic usage, and for the discovery of novel antimicrobial compounds. The first two strains have been used for the identification of BGCs for polymyxin A and E (also known as colistin) (Park et al., 2012b), respectively, and strain HS311, which possesses potent antifungal activity, has been successfully used to increase potato yield (Park et al., 2015).

It is well known that current prokaryotic species assignments in sequenced genomes in public databases may be inconsistent with correct species allocations. Recent efforts to reclassify prokaryotic species include EzBioCloud (Yoon et al., 2017), an integrated database of $16 \mathrm{~S}$ rRNA genes and genome sequences. For example, EzBioCloud version 2018.10 declared CP000154_s as a candidate species ("phylotype") for strains that were originally labeled as $P$. polymyxa (15 strains), $P$. peoriae (seven strains), and unidentified species (three strains). CP000154_s also incorporates strains E681, F4, HS311 (strains we previously isolated and sequenced) and DSM 365. Only 18 strains with the original P. polymyxa (including the type strain ATCC $842^{\mathrm{T}}$ ) and Paenibacillus species labels were classified as "genuine" $P$. polymyxa species. ANI matrix visualization of all $P$. polymyxa and CP000154_s genomes available on EzBioCloud clearly indicates that they are clustered as two separate species groups (Figure 2). Subdivision of each group into several species might be possible depending on the ANI cutoff used, but this will require further phylogenetic analysis including investigation of core and accessory genes, as well as phenotypic characterization. This implies that strains under the original species name of $P$. polymyxa $\left(38\right.$ genomes $^{3}$ ) and other closely related ones might need to be subdivided into several novel species on the basis of contemporary analysis method for genomic relatedness.

Comparative analysis of $P$. polymyxa strains has revealed that nitrogenase reductase genes ( $n$ if $H$ ) could be used to discriminate CP000154_s from genuine P. polymyxa species but not from other strains such as E681 and F4, which lack nifH genes. Although nifH was detected using PCR in P. polymyxa type strain ATCC 842 (Achouak et al., 1999), the submitted

${ }^{3}$ https://www.ncbi.nlm.nih.gov/genome/genomes/1386, as of 17 October, 2018 


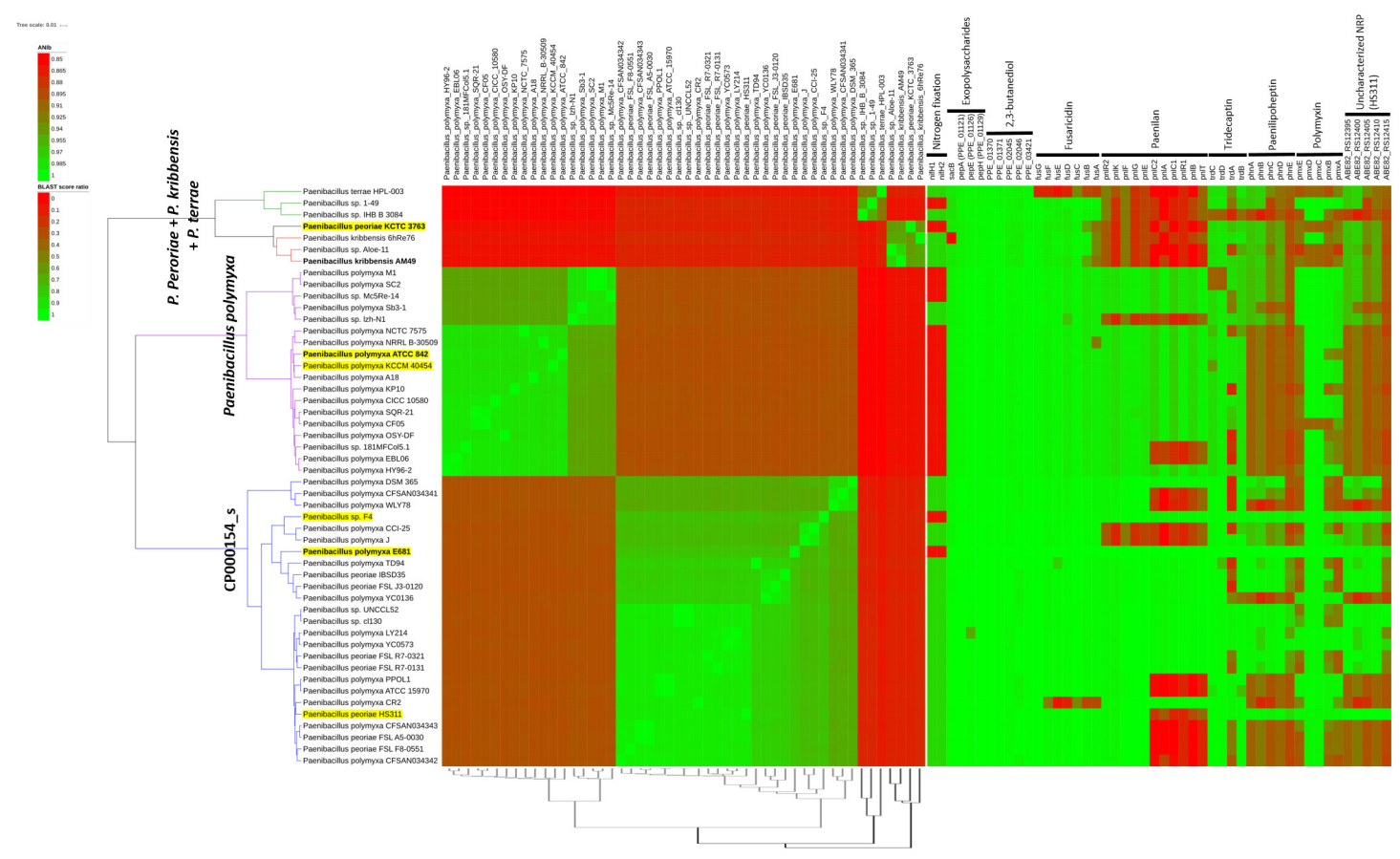

FIGURE 2 | Comparative genomic analysis of 50 selected Paenibacillus strains. All available genome sequences, identified by TrueBac ID (https://help.ezbiocloud.net/user-guide/truebac-id/about-truebac-id/) and belonging to the species P. polymyxa (18 strains), "CP000154_s" (25), P. peoriae (1), P. kribbensis (3), and P. terrae (3), were downloaded from EzBioCloud and BLASTN-based pairwise ANI values were calculated using pyani (https://github.com/widdowquinn/pyani). Species were chosen whose 16S rRNA gene sequences were similar to those of E681 (>96\% similarity). Type strains and E681 are shown in boldface; strains in a yellow background represent those sequenced by our group; and strains grouped in the same species in EzBioCloud have the same clade colors. The left heatmap shows the ANI matrix, where rows are sorted according to the complete linkage clustering data calculated in the $\mathrm{R}$ environment (hclust function) and columns are sorted as they appear in dRep (Olm et al., 2017) primary clustering (dendrogram shown at the bottom of the heatmap). The BLAST score ratio matrix shown in the right heatmap shows the presence of selected genes across strains using the LS-BSR method (Sahl et al., 2014). nifH sequences and genes starting with ABE82 were collected from $P$. durus ATCC 35681 (AJ515294.1 and AJ299453.1) and $P$. peoriae HS311 (CP011512.1), respectively. All other sequences were retrieved from strain E681. The figure was drawn using the iTOL server (Letunic and Bork, 2016).

nifH partial sequence (AJ223997) could not be found in the genome sequence of ATCC 842 (AFOX01) determined later by our group, suggesting the PCR result was an artifact. The presence and absence patterns of selected genes, responsible for nitrogen fixation, EPS production, 2,3-butanediol productions, and biosynthesis of antimicrobial compounds, are shown in the right heatmap in Figure 2. We found that genes involved in the production of EPS and 2,3-butanediol were widely distributed among the analyzed strains. It is also noteworthy that CP000154_s contains strains that have been extensively studied for plant interactions and biotechnological applications. This has also been the case for E681, DSM 365 (EPS and 2,3-butanediol production), and PPLO1 (=DSM 292). The latter strain was studied to see if it could be used as a heterologous expression system for secretory proteins because it has low exoprotease activity (Heinze et al., 2018).

\section{ANTIMICROBIAL COMPOUND PRODUCTION}

Genome sequencing of $P$. polymyxa strains revealed numerous antibiotic biosynthetic genes in the genome encoding NRPSs, polyketide synthases (PKSs), and bacteriocins. NRPS and PKS generally have a modular structure, with the modules containing multiple domains responsible for recognition, activation and condensation of an amino acid and an acyl-CoA (Aleti et al., 2015). An NRPS module typically includes at least one condensation (C) domain, an adenylation (A) domain, and a thiolation (T) domain. Other domains including those for epimerization (E) and termination (TE) domains also contribute to antibiotic synthesis. Figure 3 shows BGCs for antimicrobials characterized in strain E681. These diverse antibiotic production machineries presumably help to fight against competitors that are harmful to plants in the environment, making them useful for plant protection. In addition, some antibiotics have been used for medical purposes for the treatment of MDR bacteria. According to the antiSMASH database (Blin et al., 2017), the average number of BGCs predicted from 46 complete Paenibacillus genomes is 8.5 (11 for E681); however, this number differs depending on the strain (standard deviation $4.7 ; 1-17$ per genome). NPRS was the most common BGC among 2747 BGCs found in the sequences of all sequenced Paenibacillus genomes, including both complete and incomplete sequences. Comparison of BGC numbers among species was not meaningful because half of the analyzed genomes were from strains from unidentified 


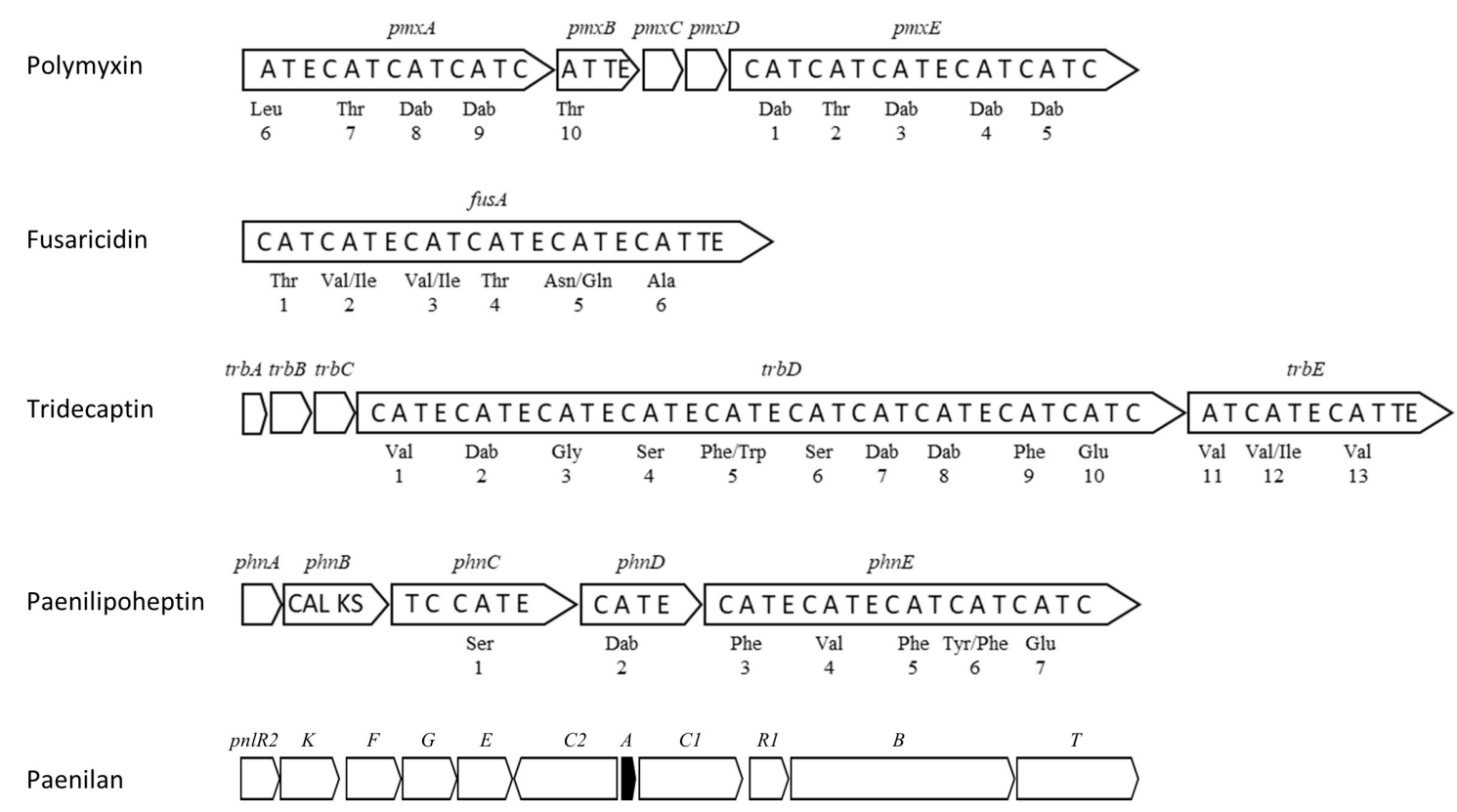

Bacillaene-like $\frac{\text { CAL TCA T KS KR ACP KS }}{\text { Gly }}$ MT ACP KS KR ACP KS ACP ACP KS DH KR ACP KS DH KR ACP KS ACP $>$ KS DH KR ACP KS DH KR MT ACP KS $>$ KR ACP KS DH KR ACP ACP KS KR

FIGURE 3 | Antibiotic gene clusters in the genome of P. polymyxa E681 (not drawn to scale). Amino acids recognized by the corresponding A-domains are shown below the modules, and the numbers indicate the order of assembly of the amino acids. The pnlA gene (black) encodes a prepeptide of paenilan. Domains are coded as follows: A, adenylation; T, thiolation; E, epimerization; C, condensation; TE, termination; CAL, CoA ligase; KS, ketosynthase; KR, ketoreductase; ACP, acyl carrier protein; MT, methyl transferase; DH, dehydratase.

Paenibacillus species. Furthermore, current species allocation needs reconsideration as mentioned earlier.

\section{Polymyxins}

\section{Identification of Polymyxin Biosynthetic Gene Cluster}

Polymyxins are cyclic heptapeptide antibiotics with fatty acidacylated tripeptide side chains that possess excellent bactericidal activity against Gram-negative bacteria. There are various types of polymyxins depending on the fatty acid type and the composition of the 3rd, 6th, 7th, and 10th amino acids. Since their discovery in 1947, more than 30 types of polymyxins have been reported (Rabanal and Cajal, 2017), including polymyxin B and $\mathrm{E}$ (colistin) that have been commonly used in clinical practice since the late 1970s for the treatment of infections caused by Gram-negative pathogenic bacteria. However, serious side effects such as severe nephrotoxicity and neurotoxicity have restricted their application to use as an ointment on local surface wounds (Hermsen et al., 2003). However, recently, limited therapeutic options for treating MDR Gram-negative pathogens have led researchers to revisit these old antibiotics as agents of last resort for fighting MDR pathogens (Chung et al., 2016).

The first NRPS gene cluster for polymyxin $(p m x)$ synthesis was identified in P. polymyxa E681 by Choi et al. (2009). This gene cluster includes five ORFs, designated $p m x A, p m x B$, $p m x C$, $p m \times D$, and $p m x E$. Analysis of PmxA (4953 amino acids), PmxB
(1102 amino acids), and PmxE (6312 amino acids) revealed four, one, and five modules, respectively, and the prediction that together they synthesize polymyxin A (Ansari et al., 2004). Polymyxin A synthesis was indeed confirmed by LC-MS analysis of wild-type E681 and pmxE knockout mutant EPT1 (Figure 4). PmxC (608 amino acids) and PmxD (577 amino acids) were proposed to be membrane transporters, and their amino acid sequences share 40.5 and $43.5 \%$ identity, respectively, with TycD and TycE members of the ABC transporter family of Brevibacillus brevis (Mootz and Marahiel, 1997). After the discovery of the polymyxin A gene cluster, several other polymyxin biosynthetic genes involved in the synthesis of polymyxins $\mathrm{B}, \mathrm{E}$, and $\mathrm{P}$ have been identified (Shaheen et al., 2011; Park et al., 2012b; Niu et al., 2013; Tambadou et al., 2015). Sequence analyses showed that polymyxin biosynthetic genes share $>90 \%$ amino acid sequence homology, and their genetic organization is highly conserved, even in other Paenibacillus species.

\section{Heterologous Expression of Polymyxin}

Restrictions on polymyxin use due to its deleterious effects on human organs have encouraged the development of lowtoxicity polymyxin derivatives. Although chemical and enzymatic modifications have been reported (Rabanal and Cajal, 2017), production of diverse derivatives has been limited by the structural complexity of polymyxins, and genetic approaches may 


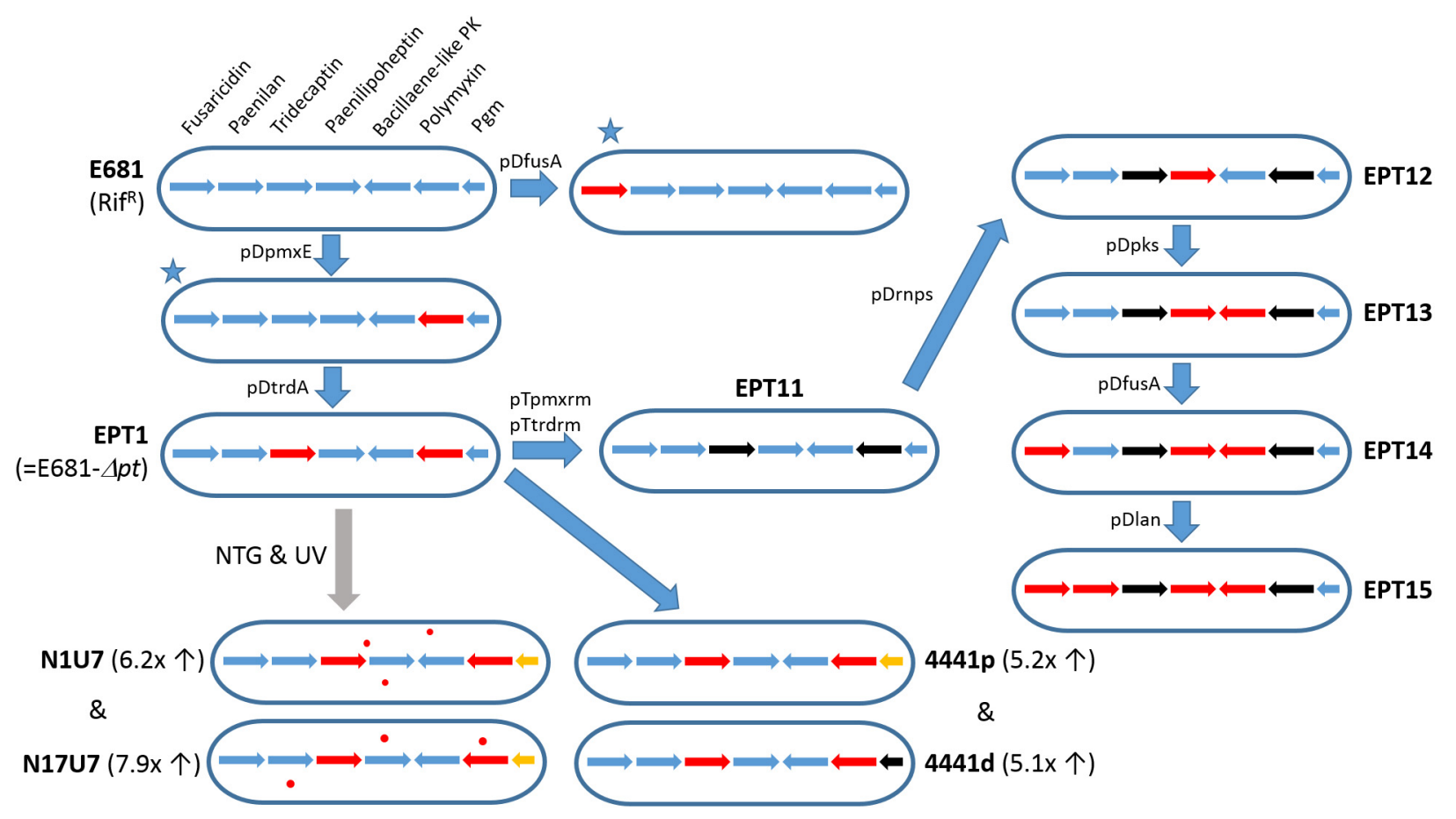

FIGURE 4 | Development of E681 derivatives for the characterization of antibiotic biosynthesis genes. Arrows indicate fusA, pnIA, trdA, phnE, PPE_03011, pmxA, and PPE_04441 (pgm) genes. Red and black arrows represent genes disrupted using knockout plasmids and genes with null mutations, respectively. The initial two fusA or pmxA knockout mutants (marked with blue stars) were constructed for functional identification of antibiotic-producing gene(s) (Choi et al., 2008, 2009). The EPT1 strain was randomly mutagenized using $N$-methyl- $N$ '-nitro- $N$-nitrosoguanidine (NTG) treatment and UV irradiation, yielding two separate mutants N1U7 and N17U7 (Kim et al., 2014). The fold increase in fusaricidin activity is shown in parentheses. Orange arrows denote Gln170Pro mutations in the pgm gene (Kim et al., 2014). Red dots represent mutations differentially occurring in each mutant strain. By introducing mutations only in the pgm gene in EPT1, fusaricidin production was enhanced (mutants 4441p and 4441d). Antibiotic biosynthetic genes in EPT11 were serially knocked out, leading to paenilan producer EPT14 (Park et al., 2017). Finally, EPT15 was constructed with no intact antibiotic genes and possessing no antimicrobial activity (Park et al., 2017).

be more successful since the gene sequences are accessible. In general, the development of new antibiotic derivatives through genetic engineering in antibiotic-producing wild-type strains is difficult due to their low transformation efficiency. For example, heterologous expression of the pmx gene cluster of $P$. polymyxa E681 has been achieved in B. subtilis 168, a well-known strain that is easy to genetically manipulate (Choi et al., 2009). The gene cluster was integrated into the amyE locus of $B$. subtilis 168 in which $s f p$ function was restored, resulting in polymyxin production only in the presence of extracellularly added L2,4-diaminobutyric acid (L-Dab), because B. subtilis does not possess a Dab synthesis mechanism and polymyxin is a Dabrich antibiotic. Dab synthesis is mediated by 2,4-diaminobutyrate aminotransferase encoded by ectB. Generally, ectB is part of the ect $A B C$ operon responsible for ectoine synthesis (Saum and Muller, 2008). However, no homologs of ectA or ect $C$ have been found in the genome of $P$. polymyxa. The genetic background suggests that Dab may not be used as an intermediate for ectoine synthesis, resulting in accumulation of Dab in the cell, which would be advantageous for polymyxin synthesis. A B. subtilis strain that could produce polymyxin without exogenous Dab was subsequently generated by introducing the ectB gene from P. polymyxa E681 (Park et al., 2012c). Additionally, expression of the pmx gene cluster in B. subtilis was completely inhibited in the $s p o 0 A$ mutant. However, in the $a b r B$ knockout and $a b r B$ spo0A double knockout organisms, polymyxin production was increased, indicating that $\mathrm{AbrB}$ represses $p m x$ gene expression. Furthermore, AbrB was confirmed to directly bind to the $p m x A$ promoter region using electrophoretic mobility shift assays.

\section{Synthesis of New Polymyxin Derivatives by Domain Swapping}

Besides chemical modification, early efforts to develop nonribosomal peptide antibiotic derivatives relied on obtaining an exogenous supply of modified amino acid precursors, endogenous engineering of precursor biosynthesis, and enzymatic tailoring such as halogenation, glycosylation, acylation, and sulfation. Since NRPSs have a modular structure, module and domain swapping methods have been used to generate novel peptide derivatives (Schneider et al., 1998; Miao et al., 2006). Recently, genetic engineering technologies such as directed evolution, synthetic biology, and CRISPR-Cas9-based gene editing have provided promising tools for the development of novel peptide antibiotics (Winn et al., 2016). However, many wild-type strains producing antibiotics are difficult to genetically manipulate, and many antibiotic genes are silent in native strains, representing bottlenecks for these genetic approaches. In a previous study, a conceptual experiment was carried out 
to generate polymyxin derivatives by domain swapping in the surrogate host B. subtilis (Kim et al., 2015). Polymyxin A produced by $P$. polymyxa E681 contains Leu-Thr in the sixth and seventh positions, while commercialized polymyxins B and E have Phe-Leu and Leu-Leu, respectively, in these positions. The authors replaced the seventh L-threonine-specific A-domain ( $\mathrm{A}_{7-\mathrm{L}-\mathrm{thr}}$-domain) region in polymyxin $\mathrm{A}$ synthetase genes with the $\mathrm{A}_{7-\mathrm{L}-\mathrm{leu}}$-domain region to construct polymyxin $\mathrm{E}$ synthetase genes. Additionally, the $\mathrm{A}_{6-\mathrm{D}-\mathrm{Leu}}$-domain region was substituted with the $\mathrm{A}_{6-\mathrm{D}-\mathrm{Phe}}$-domain region to generate polymyxin $B$ synthetase genes. Production of the engineered polymyxins in surrogate hosts was confirmed by LC-MS analysis. Thus, heterologous expression of NRPS genes in easy-to-handle hosts, and domain swapping using marker-free genome editing technologies (Jeong et al., 2015; So et al., 2017), are promising routes for the development of new antibiotic derivatives.

\section{Fusaricidins}

Fusaricidins produced by $P$. polymyxa strains are antibiotics in which six amino acids form a cyclic structure, and these compounds are highly active against Gram-positive bacteria and plant pathogenic fungi and oomycetes. Unlike other antibiotics made by $P$. polymyxa, fusaricidin activity was largely detected in the cell pellet extract, indicating association with the cell wall (Choi et al., 2008). The first fusaricidin biosynthetic gene was identified in P. polymyxa E681 as a single ORF encoding a NRPS with six modules (Choi et al., 2008). However, LCMS analysis showed that strain E681 produced various types of fusaricidins, suggesting the NRPS could produce more than one type of fusaricidin, implying that the substrate-binding pockets of A-domains may be flexible and able to bind several types of amino acids. The second, fourth, and fifth modules of the NRPS have an E-domain that generates D-amino acids, but the sixth module has no E-domain, even though the sixth amino acid of all reported fusaricidins is D-alanine. It has since been demonstrated that the A-domain of the sixth module of fusaricidin NRPS directly activates the D-form of the amino acid (Li and Jensen, 2008).

Fusaricidin production has been successfully enhanced using traditional chemical mutagenesis, but regulation of fusaricidin production has been rarely reported (Kim et al., 2014). The two mutants, N1U7 and N17U7 (Figure 4) were generated and increased fusaricidin production 6.2- and 7.9-fold, respectively, than wild type strain. Whole genome sequencing of the mutants revealed a common mutation in the pgm gene that encodes an $\alpha$-phosphoglucomutase. The pgm mutant strains exhibited reduced EPS production and increased cell growth and viability during stationary phase, which may be due to the increase in fusaricidin production in this mutant. In addition, RT-PCR analysis showed that the $p g m$ mutation increased fusaricidin gene transcription fourfold. Further studies are needed to elucidate the mechanism of this transcriptional activation.

\section{Tridecaptins}

Tridecaptins produced by Paenibacillus species are linear cationic lipopeptide antibiotics, including N-terminally acylated tridecapeptides. A gene cluster $\operatorname{tr} b A B C D E$ encoding tridecaptin synthetases was first identified in P. polymyxa E681 (Park et al., 2012a) and later in other $P$. polymyxa strains (Cochrane et al., 2015a). The $\operatorname{trb} A$ encodes a putative thioesterase, and $\operatorname{trbB}$ and trbC encode $\mathrm{ABC}$ transporters. TrbD and TrdE are NRPSs with 10 and 3 modules, respectively. Tridecaptins display strong antimicrobial activity against Gram-negative bacteria, including MRD strains of K. pneumoniae and Acinetobacter baumannii, by binding to the bacterial cell wall precursor lipid II on the inner membrane to disrupt the proton motive force (Cochrane et al., 2016). Due to low cytotoxicity and hemolytic activity, high stability in human plasma, and low levels of resistance development, tridecaptins are being studied as candidates for the treatment of MDR Gram-negative bacteria (Cochrane et al., 2014; Cochrane and Vederas, 2014). Since tridecaptins target the cell membrane, they can help other antibiotics to cross the membrane of Gram-negative bacteria in combination therapies. For example, tridecaptin has been covalently linked to other known antibiotics to enhance antimicrobial activity against Gram-negative bacteria, demonstrating great potential for effective treatment of Gram-negative pathogen infections (Cochrane et al., 2015b).

\section{Paenilipoheptin}

Paenilipoheptin was recently identified by genome mining of P. polymyxa E681. Genome analysis of E681 revealed that the paenilipoheptin BGC is composed of five ORFs encoding the hybrid NRPS-Trans-AT-PKS (Vater et al., 2018). The first two ORFs (phnAB) encode Trans-AT-PKS, and the rest (phnCDE) encode NRPSs. PhnC, PhnD, and PhnE consist of one, one, and five modules, respectively. MALDI-TOF MS analysis revealed that a C12- or C13- $\beta$-amino fatty acid is linked via its amino group to the $\mathrm{COOH}$ group of the C-terminal L-Glu of paenilipoheptin. The antimicrobial activity spectrum remains to be elucidated.

\section{Paenilan}

Paenilan, a ribosomally synthesized lantibiotic, was recently found in P. polymyxa E681 (Park et al., 2017). Analysis of the purified paenilan by nanoelectrospray ionization MS and tandem MS (MS/MS) revealed that it is a novel class I lantibiotic. The paenilan BGC spanning $13.2 \mathrm{~kb}$ comprises 11 ORFs. The prepeptide PnlA is predicted to consist of a 24 amino acid leader peptide and a 26 amino acid core peptide. A $P$. polymyxa E681 mutant (EPT14) lacking five BGCs for fusaricidin, polymyxin, tridecaptin, paenilipoheptin and uncharacterized polyketide (Figure 4) showed antimicrobial activity against Gram-positive bacteria such as B. cereus, Micrococcus luteus, and P. durus. The additional mutation in pnl BGC of EPT14 did not show antimicrobial activity against Gram-positive bacteria, indicating that the antimicrobial activity of EPT14 was derived from the paenilan.

\section{Other Antimicrobial Compounds}

Genome analysis of $P$. polymyxa E681 using antiSMASH 4.0 revealed the presence of additional, yet uncharacterized, antibiotic BGCs, including Trans-AT-PKS-OtherKS-NRPS and lasso peptide gene clusters. The Trans-AT-PKS-OtherKS-NRPS genes (PPE_03009-PPE_03012) share 78\% similarity with those 
of the bacillaene BGC of $B$. amyloliquefaciens FZB42 (Dunlap et al., 2016). The lasso peptide genes (PPE_05420) encode a putative class II bacteriocin, and $40 \%$ of genes in the cluster share similarity with those of the paeninodin BGC of P. dendritiformis C454.

\section{INDUSTRIAL IMPLICATIONS}

\section{Regulation of Sporulation}

An in-depth understanding on the regulation of $P$. polymyxa sporulation is critical for developing industrial applications based on this strain, especially as continuous cultivation prohibits sporulation during the fermentation process. Sporulation initiation in Bacillus and related organisms including Paenibacillus spp. is dependent on a well-conserved multicomponent phosphorelay system. Multiple histidine kinases sense environmental sporulation signals and autophosphorylate themselves. The phosphate group of the histidine kinases is transferred to Spo0F, then to Spo0B, and finally to Spo0A. Phosphorylated Spo0A regulates the expression of genes related to sporulation. In general, $\mathrm{Spo} 0 \mathrm{~F}$ and $\mathrm{Spo} 0 \mathrm{~A}$ are highly conserved in Bacillus species, while SpoOB and the histidine kinases are less and poorly conserved, respectively. Spo0F and Spo0A of $P$. polymyxa E681 share 73 and 68\% amino acid sequence identity, respectively, with those of B. subtilis 168, while Spo0B of E681 shares only $14 \%$ identity with B. subtilis Spo0B. The $P$. polymyxa E681 genome does not contain sporulation histidine kinase homologs to those in B. subtilis, although putative sporulation histidine kinase genes have been identified (Park et al., 2012e). Specifically, a histidine kinase (Kin1377) from $P$. polymyxa successfully restored sporulation in a sporulationdeficient $B$. subtilis mutant in which two major histidine kinase genes, $k i n A$ and $\operatorname{kin} B$, were knocked out. The result suggested that the Kin1377 may be a sporulation histidine kinase in $P$. polymyxa. Another interesting sporulation histidine kinase (Kin1038) was also identified that partially restored sporulation in a $\operatorname{kin} A$ and $\operatorname{kin} B$ double mutant of $B$. subtilis. However, the additional introduction of the $P$. polymyxa spo0A gene into the mutant strain harboring Kin1038 induced significantly more spore formation. These results suggest that Kin1038 can bypass Spo0F and Spo0B, and transfer the phosphate group directly to Spo0A. A similar direct transfer of phosphate from histidine kinases to Spo0A was found in Clostridium species lacking $s p o 0 F$ or $s p o 0 B$ homologs in the genome (Worner et al., 2006).

\section{Active Inclusion Body Formation}

Overexpression of recombinant proteins in $E$. coli often results in inclusion bodies in which misfolded proteins are aggregated and biologically inactive. This is commercially disadvantageous because solubilization of the inclusion bodies and refolding of the proteins are costly. Therefore, the formation of biologically active inclusion bodies in $E$. coli is attractive from a commercial perspective. $P$. polymyxa pyruvate oxidase (PoxB) forms large inclusion bodies when overexpressed in E. coli (Park et al., 2012d), which generates inclusion bodies with pyruvate oxidase activity. Fusion of GFP and B. subtilis AmyE to the PoxB also induced the formation of biologically active aggregates. The results indicate that $\mathrm{PoxB}$ can be used as a fusion partner for the formation of active inclusion bodies in E. coli.

\section{Boosting the Strength of Cement Paste}

One interesting study demonstrated the industrial applicability of strain E681 (Park et al., 2014). Cement paste containing strain E681 displayed strong antifungal activity against Aspergillus niger, a deleterious fungus commonly found in cement buildings and structures. Strain E681 was also shown to help repair cracks by precipitation of calcium carbonate crystals in cement paste. These results suggest that strain E681 could be used in the development of multifunctional cement mortar.

\section{CONCLUSION}

Herein, we summarized literature demonstrating that information derived from previous studies on $P$. polymyxa E681 provide an important gateway for promoting plant health and human healthcare. Strain E681 was initially studied as an outstanding PGPR based on its potent plant growth-promoting and plant-protecting activities, and bioinformatic and functional analyses of its genome subsequently illuminated various characteristics that benefit plants. More recently, strain E681 was found to be adept at producing various antibiotics, most notably polymyxins, recently re-instated as a therapy of last resort for treating Gram-negative infections. Other antibiotics produced by E681 include tridecaptin, paenilan, paenilipoheptin, and a putative polyketide, all of which are worthy of further study for the development of novel antibiotics against MDR human pathogenic bacteria. Fusaricidins elicit strong ISR effects, making them attractive as plant protectant agents in agriculture. A recombinant E681 strain that produces only fusaricidin at a high level by knocking out BGCs for other antimicrobial compounds has been reported.

We propose the need to reclassify sequenced strains in publicly available databases under the original designation of $P$. polymyxa species using genomic analysis of strain E681 and its close relatives. This could widen our genomic perspective on $P$. polymyxa and related species that have evolved under specific environmental niches, especially regarding their interactions with other organisms such as plants. In conclusion, bringing together up-to-date knowledge on the versatile $P$. polymyxa E681 strain could assist the development of highly functional microbial fertilizers and biocontrol agents, as well as industrial strains for the production of antibiotics and other valuable metabolites.

\section{AUTHOR CONTRIBUTIONS}

HJ conceived the project. HJ and S-HP sequenced the genomes and conducted comparative analysis. All authors wrote the manuscript and reviewed the final version of the manuscript prior to submission for publication. 


\section{FUNDING}

This research was supported by the Korea Research Institute of Bioscience and Biotechnology (KRIBB) Initiative Program, the Bio \& Medical Technology Development Program of the National Research Foundation (NRF) funded by the

\section{REFERENCES}

Achouak, W., Normand, P., and Heulin, T. (1999). Comparative phylogeny of rrs and nifH genes in the Bacillaceae. Int. J. Syst. Bacteriol. 49(Pt 3), 961-967. doi: 10.1099/00207713-49-3-961

Aleti, G., Sessitsch, A., and Brader, G. (2015). Genome mining: prediction of lipopeptides and polyketides from Bacillus and related Firmicutes. Comput. Struct. Biotechnol. J. 13, 192-203. doi: 10.1016/j.csbj.2015.03.003

Ansari, M. Z., Yadav, G., Gokhale, R. S., and Mohanty, D. (2004). NRPS-PKS: a knowledge-based resource for analysis of NRPS/PKS megasynthases. Nucleic Acids Res. 32, W405-W413. doi: 10.1093/nar/gkh359

Ash, C., Farrow, J. A. E., Wallbanks, S., and Collins, M. D. (1991). Phylogenetic heterogeneity of the genus Bacillus revealed by comparative analysis of smallsubunit-ribosomal RNA sequences. Lett. Appl. Microbiol. 13, 202-206. doi: 10. 1111/j.1472-765X.1991.tb00608.x

Ash, C., Priest, F. G., and Collins, M. D. (1993). Molecular identification of rRNA group 3 bacilli (Ash, Farrow, Wallbanks and Collins) using a PCR probe test. Proposal for the creation of a new genus Paenibacillus. Antonie Van Leeuwenhoek 64, 253-260. doi: 10.1007/BF00873085

Bach, E., Seger, G. D. D. S., Fernandes, G. D. C., Lisboa, B. B., and Passaglia, L. M. P. (2016). Evaluation of biological control and rhizosphere competence of plant growth promoting bacteria. Appl. Soil Ecol. 99, 141-149. doi: 10.1016/j.apsoil. 2015.11.002

Bakker, P. A., Pieterse, C. M., and van Loon, L. C. (2007). Induced systemic resistance by fluorescent Pseudomonas spp. Phytopathology 97, 239-243. doi: 10.1094/PHYTO-97-2-0239

Bal, A., and Chanway, C. P. (2012a). 15N foliar dilution of western red cedar in response to seed inoculation with diazotrophic Paenibacillus polymyxa. Biol. Fertil. Soils 48, 967-971. doi: 10.1007/s00374-0120699-9

Bal, A., and Chanway, C. P. (2012b). Evidence of nitrogen fixation in lodgepole pine inoculated with diazotrophic Paenibacillus polymyxa. Botany 90, 891-896. doi: 10.1139/b2012-044

Berendsen, R. L., Pieterse, C. M., and Bakker, P. A. (2012). The rhizosphere microbiome and plant health. Trends Plant Sci. 17, 478-486. doi: 10.1016/j. tplants.2012.04.001

Blin, K., Medema, M. H., Kottmann, R., Lee, S. Y., and Weber, T. (2017). The antiSMASH database, a comprehensive database of microbial secondary metabolite biosynthetic gene clusters. Nucleic Acids Res. 45, D555-D559. doi: 10.1093/nar/gkw960

Cheong, H., Park, S. Y., Ryu, C. M., Kim, J. F., and Park, C. S. (2005). Diversity of root-associated Paenibacillus spp. in winter crops from southern part of Korea. J. Microbiol. Biotechnol. 16, 1286-1298.

Choi, O., Kim, J., Ryu, C. M., and Park, C. S. (2004). Colonization and population changes of a biocontrol agent, Paenibacillus polymyxa E681, in seeds and roots. Plant Pathol. J. 20, 97-102.

Cho, Y. S., Park, C. S., Lee, S. G., Lee, Y. G., Lim, C. G., Cha, J. S., et al. (1999). Bacterial Plant Pathology. Seoul: Seoul National University Press.

Choi, S. K., Park, S. Y., Kim, R., Kim, S. B., Lee, C. H., Kim, J. F., et al. (2009). Identification of a polymyxin synthetase gene cluster of Paenibacillus polymyxa and heterologous expression of the gene in Bacillus subtilis. J. Bacteriol. 191, 3350-3358. doi: 10.1128/JB.01728-08

Choi, S. K., Park, S. Y., Kim, R., Lee, C. H., Kim, J. F., and Park, S. H. (2008). Identification and functional analysis of the fusaricidin biosynthetic gene of Paenibacillus polymyxa E681. Biochem. Biophys. Res. Commun. 365, 89-95. doi: 10.1016/j.bbrc.2007.10.147

Chung, J. H., Bhat, A., Kim, C. J., Yong, D., and Ryu, C. M. (2016). Combination therapy with polymyxin $\mathrm{B}$ and netropsin against clinical
Korean Government (MSIT) (NRF-2018M3A9F3079565), and the Strategic Initiative for Microbiomes in Agriculture and Food, Ministry of Agriculture, Food and Rural Affairs, South Korea [as part of the (multi-ministerial) Genome Technology to Business Translation Program 918017-4], South Korea.

isolates of multidrug-resistant Acinetobacter baumannii. Sci. Rep. 6:28168. doi: $10.1038 /$ srep 28168

Cochrane, S. A., Findlay, B., Bakhtiary, A., Acedo, J. Z., Rodriguez-Lopez, E. M., Mercier, P., et al. (2016). Antimicrobial lipopeptide tridecaptin $A_{1}$ selectively binds to Gram-negative lipid II. Proc. Natl. Acad. Sci. U.S.A. 113, 11561-11566. doi: 10.1073/pnas.1608623113

Cochrane, S. A., Li, X., He, S., Yu, M., Wu, M., and Vederas, J. C. (2015a). Synthesis of tridecaptin-antibiotic conjugates with in vivo activity against Gramnegative bacteria. J. Med. Chem. 58, 9779-9785. doi: 10.1021/acs.jmedchem. 5 b01578

Cochrane, S. A., Lohans, C. T., van Belkum, M. J., Bels, M. A., and Vederas, J. C. (2015b). Studies on tridecaptin B(1), a lipopeptide with activity against multidrug resistant Gram-negative bacteria. Org. Biomol. Chem. 13, 6073-6081. doi: $10.1039 / \mathrm{c} 50$ ob00780a

Cochrane, S. A., Lohans, C. T., Brandelli, J. R., Mulvey, G., Armstrong, G. D., and Vederas, J. C. (2014). Synthesis and structure-activity relationship studies of N-terminal analogues of the antimicrobial peptide tridecaptin A(1). J. Med. Chem. 57, 1127-1131. doi: 10.1021/jm401779d

Cochrane, S. A., and Vederas, J. C. (2014). Unacylated tridecaptin A(1) acts as an effective sensitiser of Gram-negative bacteria to other antibiotics. Int. J. Antimicrob. Agents 44, 493-499. doi: 10.1016/j.ijantimicag.2014. 08.008

Cochrane, S. A., and Vederas, J. C. (2016). Lipopeptides from Bacillus and Paenibacillus spp.: a gold mine of antibiotic candidates. Med. Res. Rev. 36, 4-31. doi: $10.1002 /$ med.21321

Crisp, P. A., Ganguly, D., Eichten, S. R., Borevitz, J. O., and Pogson, B. J. (2016). Reconsidering plant memory: intersections between stress recovery, RNA turnover, and epigenetics. Sci. Adv. 2:e1501340. doi: 10.1126/sciadv. 1501340

Dunlap, C. A., Kim, S. J., Kwon, S. W., and Rooney, A. P. (2016). Bacillus velezensis is not a later heterotypic synonym of Bacillus amyloliquefaciens; Bacillus methylotrophicus, Bacillus amyloliquefaciens subsp. plantarum and 'Bacillus oryzicola' are later heterotypic synonyms of Bacillus velezensis based on phylogenomics. Int. J. Syst. Evol. Microbiol. 66, 1212-1217. doi: 10.1099/ijsem. 0.000858

Eastman, A. W., Heinrichs, D. E., and Yuan, Z. C. (2014). Comparative and genetic analysis of the four sequenced Paenibacillus polymyxa genomes reveals a diverse metabolism and conservation of genes relevant to plant-growth promotion and competitiveness. BMC Genomics 15:851. doi: 10.1186/1471-216415-851

Emmert, E. A., and Handelsman, J. (1999). Biocontrol of plant disease: a (Gram-) positive perspective. FEMS Microbiol. Lett. 171, 1-9. doi: 10.1111/j.1574-6968. 1999.tb13405.x

Farag, M. A., Zhang, H., and Ryu, C. M. (2013). Dynamic chemical communication between plants and bacteria through airborne signals: induced resistance by bacterial volatiles. J. Chem. Ecol. 39, 1007-1018. doi: 10.1007/s10886-0130317-9

Fu, J., and Wang, S. (2011). Insights into auxin signaling in plant-pathogen interactions. Front. Plant Sci. 2:74. doi: 10.3389/fpls.2011.00074

Garg, S. K., and Jain, A. (1995). Fermentative production of 2,3-butanediol: a review. Bioresour. Technol. 51, 103-109. doi: 10.1016/0960-8524(94) 00136-O

Goris, J., Konstantinidis, K. T., Klappenbach, J. A., Coenye, T., Vandamme, P., and Tiedje, J. M. (2007). DNA-DNA hybridization values and their relationship to whole-genome sequence similarities. Int. J. Syst. Evol. Microbiol. 57(Pt 1), 81-91. doi: 10.1099/ijs.0.64483-0

Grady, E. N., MacDonald, J., Liu, L., Richman, A., and Yuan, Z. C. (2016). Current knowledge and perspectives of Paenibacillus: 
a review. Microb. Cell Fact. 15:203. doi: 10.1186/s12934-0160603-7

Haggag, W. M. (2007). Colonization of exopolysaccharide-producing Paenibacillus polymyxa on peanut roots for enhancing resistance against crown rot disease. Afr. J. Biotechnol. 6, 1568-1577.

Hahm, M. S., Sumayo, M., Hwang, Y. J., Jeon, S. A., Park, S. J., Lee, J. Y., et al. (2012). Biological control and plant growth promoting capacity of rhizobacteria on pepper under greenhouse and field conditions. J. Microbiol. 50, 380-385. doi: 10.1007/s12275-012-1477-y

Heinze, S., Zimmermann, K., Ludwig, C., Heinzlmeir, S., Schwarz, W. H., Zverlov, V. V., et al. (2018). Evaluation of promoter sequences for the secretory production of a Clostridium thermocellum cellulase in Paenibacillus polymyxa. Appl. Microbiol. Biotechnol. 102, 10147-10159. doi: 10.1007/s00253-0189369-7

Hermsen, E. D., Sullivan, C. J., and Rotschafer, J. C. (2003). Polymyxins: pharmacology, pharmacokinetics, pharmacodynamics, and clinical applications. Infect. Dis. Clin. North Am. 17, 545-562. doi: 10.1016/S08915520(03)00058-8

Heulin, T., Berge, O., Mavingui, P., Gouzou, L., Hebbar, K. P., and Balandreau, J. (1994). Bacillus polymyxa and Rahnella aquatilis, the dominant N2-fixing bacteria associated with wheat rhizosphere in French soils. Eur. J. Soil Biol. 30, 35-42.

Huang, Z., Zhao, F., and Li, Y. H. (2017). Isolation of Paenibacillus tumbae sp. nov., from the tomb of the emperor Yang of the Sui dynasty, and emended description of the genus Paenibacillus. Antonie Van Leeuwenhoek 110, 357-364. doi: 10.1007/s10482-016-0807-1

Jeong, D. E., Park, S. H., Pan, J. G., Kim, E. J., and Choi, S. K. (2015). Genome engineering using a synthetic gene circuit in Bacillus subtilis. Nucleic Acids Res. 43:e42. doi: 10.1093/nar/gku1380

Jeong, H., Choi, S. K., Park, S. Y., Kim, S. H., and Park, S. H. (2012). Draft genome sequence of Paenibacillus peoriae strain KCTC $3763^{\mathrm{T}}$. J. Bacteriol. 194, 1237-1238. doi: 10.1128/JB.06577-11

Jeong, H., Kim, J. F., Park, Y.-K., Kim, S.-B., Kim, C., and Park, S.-H. (2006). Genome snapshot of Paenibacillus polymyxa ATCC $842^{\mathrm{T}}$. J. Microbiol. Biotechnol. 16, 1650-1655.

Jeong, H., Park, S. Y., Chung, W. H., Kim, S. H., Kim, N., Park, S. H., et al. (2011). Draft genome sequence of the Paenibacillus polymyxa type strain (ATCC $842^{\mathrm{T}}$ ), a plant growth-promoting bacterium. J. Bacteriol. 193, 5026-5027. doi: 10.1128/ JB.05447-11

Jeong, H., Yoon, S. H., Yu, D. S., Oh, T. K., and Kim, J. F. (2008). Recent progress of microbial genome projects in Korea. Biotechnol. J. 3, 601-611. doi: 10.1002/ biot. 200800016

Jung, S. G., Jang, J. H., Kim, A. Y., Lim, M. C., Kim, B., Lee, J., et al. (2013). Removal of pathogenic factors from 2,3-butanediol-producing Klebsiella species by inactivating virulence-related wabG gene. Appl. Microbiol. Biotechnol. 97, 1997007. doi: 10.1007/s00253-012-4284-9

Kim, B., Song, G. C., and Ryu, C. M. (2016). Root exudation by aphid leaf infestation recruits root-associated Paenibacillus spp. to lead plant insect susceptibility. J. Microbiol. Biotechnol. 26, 549-557. doi: 10.4014/jmb.1511. 11058

Kim, H. R., Park, S. Y., Kim, S. B., Jeong, H., Choi, S. K., and Park, S. H. (2014). Inactivation of the phosphoglucomutase gene pgm in Paenibacillus polymyxa leads to overproduction of fusaricidin. J. Ind. Microbiol. Biotechnol. 41, 1405-1414. doi: 10.1007/s10295-014-1470-Z

Kim, J. F., Jeong, H., Park, S. Y., Kim, S. B., Park, Y. K., Choi, S. K., et al. (2010). Genome sequence of the polymyxin-producing plant-probiotic rhizobacterium Paenibacillus polymyxa E681. J. Bacteriol. 192, 6103-6104. doi: 10.1128/JB. 00983-10

Kim, S. Y., Park, S. Y., Choi, S. K., and Park, S. H. (2015). Biosynthesis of polymyxins $\mathrm{B}, \mathrm{E}$, and $\mathrm{P}$ using genetically engineered polymyxin synthetases in the surrogate host Bacillus subtilis. J. Microbiol. Biotechnol. 25, 1015-1025. doi: 10.4014/jmb.1505.05036

Kloepper, J. W., Ryu, C. M., and Zhang, S. (2004). Induced systemic resistance and promotion of plant growth by Bacillus spp. Phytopathology 94, 1259-1266. doi: 10.1094/PHYTO.2004.94.11.1259

Kunst, F., Ogasawara, N., Moszer, I., Albertini, A. M., Alloni, G., Azevedo, V., et al. (1997). The complete genome sequence of the Gram-positive bacterium Bacillus subtilis. Nature 390, 249-256. doi: 10.1038/36786
Kwon, Y. S., Lee, D. Y., Rakwal, R., Baek, S. B., Lee, J. H., Kwak, Y. S., et al. (2016). Proteomic analyses of the interaction between the plant-growth promoting rhizobacterium Paenibacillus polymyxa E681 and Arabidopsis thaliana. Proteomics 16, 122-135. doi: 10.1002/pmic.201500196

Lee, B., Farag, M. A., Park, H. B., Kloepper, J. W., Lee, S. H., and Ryu, C. M. (2012). Induced resistance by a long-chain bacterial volatile: elicitation of plant systemic defense by a C13 volatile produced by Paenibacillus polymyxa. PLoS One 7:e48744. doi: 10.1371/journal.pone.0048744

Letunic, I., and Bork, P. (2016). Interactive tree of life (iTOL) v3: an online tool for the display and annotation of phylogenetic and other trees. Nucleic Acids Res. 44, W242-W245. doi: 10.1093/nar/gkw290

Levy, M., Thaiss, C. A., and Elinav, E. (2016). Metabolites: messengers between the microbiota and the immune system. Genes Dev. 30, 1589-1597. doi: 10.1101/ gad.284091.116

Li, H., Ruan, J., and Durbin, R. (2008). Mapping short DNA sequencing reads and calling variants using mapping quality scores. Genome Res. 18, 1851-1858. doi: $10.1101 /$ gr.078212.108

Li, J., and Jensen, S. E. (2008). Nonribosomal biosynthesis of fusaricidins by Paenibacillus polymyxa PKB1 involves direct activation of a $\mathrm{D}$-amino acid. Chem. Biol. 15, 118-127. doi: 10.1016/j.chembiol.2007.12.014

Lugtenberg, B., and Kamilova, F. (2009). Plant-growth-promoting rhizobacteria. Annu. Rev. Microbiol. 63, 541-556. doi: 10.1146/annurev.micro.62.081307. 162918

Ma, M., Wang, C., Ding, Y., Li, L., Shen, D., Jiang, X., et al. (2011). Complete genome sequence of Paenibacillus polymyxa SC2, a strain of plant growth-promoting Rhizobacterium with broad-spectrum antimicrobial activity. J. Bacteriol. 193, 311-312. doi: 10.1128/JB.01234-10

Miao, V., Coeffet-Le Gal, M. F., Nguyen, K., Brian, P., Penn, J., Whiting, A., et al. (2006). Genetic engineering in Streptomyces roseosporus to produce hybrid lipopeptide antibiotics. Chem. Biol. 13, 269-276. doi: 10.1016/j.chembiol.2005. 12.012

Mootz, H. D., and Marahiel, M. A. (1997). The tyrocidine biosynthesis operon of Bacillus brevis: complete nucleotide sequence and biochemical characterization of functional internal adenylation domains. J. Bacteriol. 179, 6843-6850. doi: 10.1128/jb.179.21.6843-6850.1997

Mus, F., Alleman, A. B., Pence, N., Seefeldt, L. C., and Peters, J. W. (2018). Exploring the alternatives of biological nitrogen fixation. Metallomics 10, 523538. doi: $10.1039 / \mathrm{c} 8 \mathrm{mt} 00038 \mathrm{~g}$

Niu, B., Rueckert, C., Blom, J., Wang, Q., and Borriss, R. (2011). The genome of the plant growth-promoting rhizobacterium Paenibacillus polymyxa M-1 contains nine sites dedicated to nonribosomal synthesis of lipopeptides and polyketides. J. Bacteriol. 193, 5862-5863. doi: 10.1128/JB.05806-11

Niu, B., Vater, J., Rueckert, C., Blom, J., Lehmann, M., Ru, J. J., et al. (2013). Polymyxin $\mathrm{P}$ is the active principle in suppressing phytopathogenic Erwinia spp. by the biocontrol rhizobacterium Paenibacillus polymyxa M-1. BMC Microbiol. 13:137. doi: 10.1186/1471-2180-13-137

Okonkwo, C. C., Ujor, V., and Ezeji, T. (2018). Molecular inactivation of exopolysaccharide biosynthesis in Paenibacillus polymyxa DSM 365 for enhanced 2,3-butanediol production. bioRxiv [Preprint]. doi: 10.1101/331843

Olm, M. R., Brown, C. T., Brooks, B., and Banfield, J. F. (2017). dRep: a tool for fast and accurate genomic comparisons that enables improved genome recovery from metagenomes through de-replication. ISME J. 11, 2864-2868. doi: 10.1038 /ismej.2017.126

Ongena, M., and Jacques, P. (2008). Bacillus lipopeptides: versatile weapons for plant disease biocontrol. Trends Microbiol. 16, 115-125. doi: 10.1016/j.tim.2007. 12.009

Padda, K. P., Puri, A., Zeng, Q., and Chanway, C. P. (2017). Effect of GFP-tagging on nitrogen fixation and plant growth promotion of an endophytic diazotrophic strain of Paenibacillus polymyxa. Botany 95, 933-942. doi: 10.1139/cjb-20170056

Palaniyandi, S. A., Yang, S. H., Zhang, L., and Suh, J. W. (2013). Effects of actinobacteria on plant disease suppression and growth promotion. Appl. Microbiol. Biotechnol. 97, 9621-9636. doi: 10.1007/s00253-013-5206-1

Park, H. B., Lee, B., Kloepper, J. W., and Ryu, C. M. (2013). One shot-two pathogens blocked: exposure of Arabidopsis to hexadecane, a long chain volatile organic compound, confers induced resistance against both Pectobacterium carotovorum and Pseudomonas syringae. Plant Signal. Behav. 8, e24619. doi: $10.4161 /$ psb. 24619 
Park, J. E., Kim, H. R., Park, S. Y., Choi, S. K., and Park, S. H. (2017). Identification of the biosynthesis gene cluster for the novel lantibiotic paenilan from Paenibacillus polymyxa $\mathrm{E} 681$ and characterization of its product. J. Appl. Microbiol. 123, 1133-1147. doi: 10.1111/jam.13580

Park, K. Y., Yeon Seo, S., Oh, B.-R., Seo, J.-W., and Jung Kim, Y. (2018). 2,3-butanediol induces systemic acquired resistance in the plant immune response. J. Plant Biol. 61, 424-434. doi: 10.1007/s12374-0180421-z

Park, S. H., Choi, S. K., Kim, J. F., Ryu, C. M., and Park, S. Y. (2012a). Tridecaptin synthetase and gene thereof. Republic of Korea Patent No 10-1165247. Daejeon: K.I.P. Office.

Park, S. H., Kim, J. F., Lee, C. H., Choi, S. K., Jeong, H., Kim, S. B., et al. (2012b). Polymyxin synthetase and gene cluster thereof. U.S. Patent No 8,329,430. Washington, DC: U.S.P.A.T. Office.

Park, S. Y., Choi, S. K., Kim, J., Oh, T. K., and Park, S. H. (2012c). Efficient production of polymyxin in the surrogate host Bacillus subtilis by introducing a foreign $e c t B$ gene and disrupting the $a b r B$ gene. Appl. Environ. Microbiol. 78, 4194-4199. doi: 10.1128/AEM.07912-11

Park, S. Y., Park, S. H., and Choi, S. K. (2012d). Active inclusion body formation using Paenibacillus polymyxa PoxB as a fusion partner in Escherichia coli. Anal. Biochem. 426, 63-65. doi: 10.1016/j.ab.2012. 04.002

Park, S. Y., Park, S. H., and Choi, S. K. (2012e). Characterization of sporulation histidine kinases of Paenibacillus polymyxa. Res. Microbiol. 163, 272-278. doi: 10.1016/j.resmic.2012.02.003

Park, S. H., Choi, S. K., Park, S. Y., Jeon, J. H., Kim, H. R., and Kim, Y. T. (2015). Novel Paenibacillus sp. and the method for yield of potato using the same. Republic of Korea Patent No 10-1498155. Daejeon: K.I.P. Office.

Park, S. J., Park, S. H., and Ghim, S. Y. (2014). The effects of Paenibacillus polymyxa E681 on antifungal and crack remediation of cement paste. Curr. Microbiol. 69, 412-416. doi: 10.1007/s00284-014-0604-x

Park, S. Y., Kim, R., Ryu, C. M., Choi, S. K., Lee, C. H., Kim, J. G., et al. (2008). Citrinin, a mycotoxin from Penicillium citrinum, plays a role in inducing motility of Paenibacillus polymyxa. FEMS Microbiol. Ecol. 65, 229-237. doi: 10.1111/j.1574-6941.2008.00492.x

Phi, Q. T., Oh, S. H., Park, Y. M., Park, S. H., Ryu, C. M., and Ghim, S. Y. (2008a). Isolation and characterization of transposon-insertional mutants from Paenibacillus polymyxa E681 altering the biosynthesis of indole-3-acetic acid. Curr. Microbiol. 56, 524-530. doi: 10.1007/s00284-0089118-8

Phi, Q. T., Park, Y. M., Ryu, C. M., Park, S. H., and Ghim, S. Y. (2008b). Functional identification and expression of indole-3-pyruvate decarboxylase from Paenibacillus polymyxa E681. J. Microbiol. Biotechnol. 18, 1235-1244.

Postler, T. S., and Ghosh, S. (2017). Understanding the holobiont: how microbial metabolites affect human health and shape the immune system. Cell Metab. 26, 110-130. doi: 10.1016/j.cmet.2017.05.008

Rabanal, F., and Cajal, Y. (2017). Recent advances and perspectives in the design and development of polymyxins. Nat. Prod. Rep. 34, 886-908. doi: 10.1039/ c7np00023e

Raza, W., Yang, R., and Shen, Q.-R. (2008). Paenibacillus polymyxa: antibiotic, hydrolytic enzymes and hazard assessment. J. Plant Pathol. 90, 419-430.

Richter, M., and Rossello-Mora, R. (2009). Shifting the genomic gold standard for the prokaryotic species definition. Proc. Natl. Acad. Sci. U.S.A. 106, 1912619131. doi: 10.1073/pnas.0906412106

Ruschel, A., Henis, Y., and Salati, E. (1975). Nitrogen-15 tracing of N-fixation with soil-grown sugarcane seedlings. Soil Biol. Biochem. 7, 181-182. doi: 10.1016/ 0038-0717(75)90017-6

Rütering, M., Cress, B. F., Schilling, M., Rühmann, B., Koffas, M. A. G., Sieber, V., et al. (2017). Tailor-made exopolysaccharides-CRISPR-Cas9 mediated genome editing in Paenibacillus polymyxa. Synth. Biol. 2:ysx007. doi: 10.1093/synbio/ ysx007

Rütering, M., Schmid, J., Gansbiller, M., Braun, A., Kleinen, J., Schilling, M., et al. (2018). Rheological characterization of the exopolysaccharide Paenan in surfactant systems. Carbohydr. Polym. 181, 719-726. doi: 10.1016/j.carbpol. 2017.11.086

Ryu, C.-M., Kim, J., Choi, O., Kim, S. H., and Park, C. S. (2006). Improvement of biological control capacity of Paenibacillus polymyxa E681 by seed pelleting on sesame. Biol. Control 39, 282-289. doi: 10.1016/j.biocontrol.2006.04.014
Ryu, C. M., Hu, C.-H., Locy, R. D., and Kloepper, J. W. (2005a). Study of mechanisms for plant growth promotion elicited by rhizobacteria in Arabidopsis thaliana. Plant Soil 268, 285-292.

Ryu, C.-M., Kim, J., Choi, O., Park, S.-Y., Park, S.-H., and Park, C. S. (2005b). Nature of a root-associated Paenibacillus polymyxa from field-grown winter barley in Korea. J. Microbiol. Biotechnol. 15, 984-991.

Ryu, C. M., and Park, C. S. (1997). "Enhancement of plant growth induced by endospore forming PGPR strain, Bacillus polymyxa E681," in Plant GrowthPromoting Rhizobacteria - Present Status and Future Prospects, eds A. Ogoshi, K. Kobayashi, Y. Homma, F. Komada, N. Knodo, and S. Akino (Sapporo: Nakanishi Printing), 186-190.

Sahl, J. W., Caporaso, J. G., Rasko, D. A., and Keim, P. (2014). The large-scale blast score ratio (LS-BSR) pipeline: a method to rapidly compare genetic content between bacterial genomes. PeerJ 2:e332. doi: 10.7717/peerj.332

Saum, S. H., and Muller, V. (2008). Growth phase-dependent switch in osmolyte strategy in a moderate halophile: ectoine is a minor osmolyte but major stationary phase solute in Halobacillus halophilus. Environ. Microbiol. 10, 716726. doi: 10.1111/j.1462-2920.2007.01494.x

Schmitz, R. A., Klopprogge, K., and Grabbe, R. (2002). Regulation of nitrogen fixation in Klebsiella pneumoniae and Azotobacter vinelandii: NifL, transducing two environmental signals to the nif transcriptional activator NifA. J. Mol. Microbiol. Biotechnol. 4, 235-242.

Schneider, A., Stachelhaus, T., and Marahiel, M. A. (1998). Targeted alteration of the substrate specificity of peptide synthetases by rational module swapping. Mol. Gen. Genet. 257, 308-318. doi: 10.1007/s004380050652

Seul, K. J., Park, S. H., Ryu, C. M., Lee, Y. H., and Ghim, S. Y. (2007). Proteome analysis of Paenibacillus polymyxa E681 affected by barley. J. Microbiol. Biotechnol. 17, 934-944.

Shaheen, M., Li, J., Ross, A. C., Vederas, J. C., and Jensen, S. E. (2011). Paenibacillus polymyxa $\mathrm{PKB} 1$ produces variants of polymyxin B-type antibiotics. Chem. Biol. 18, 1640-1648. doi: 10.1016/j.chembiol.2011.09.017

Sharifi, R., and Ryu, C. M. (2018). Revisiting bacterial volatile-mediated plant growth promotion: lessons from the past and objectives for the future. Ann. Bot. 122, 349-358. doi: 10.1093/aob/mcy108

So, Y., Park, S. Y., Park, E. H., Park, S. H., Kim, E. J., Pan, J. G., et al. (2017). A highly efficient CRISPR-Cas9-mediated large genomic deletion in Bacillus subtilis. Front. Microbiol. 8:1167. doi: 10.3389/fmicb.2017.01167

Steenhoudt, O., and Vanderleyden, J. (2000). Azospirillum, a free-living nitrogenfixing bacterium closely associated with grasses: genetic, biochemical and ecological aspects. FEMS Microbiol. Rev. 24, 487-506. doi: 10.1111/j.1574-6976. 2000.tb00552.x

Storm, D. R., Rosenthal, K. S., and Swanson, P. E. (1977). Polymyxin and related peptide antibiotics. Annu. Rev. Biochem. 46, 723-763. doi: 10.1146/annurev.bi. 46.070177.003451

Sumi, C. D., Yang, B. W., Yeo, I. C., and Hahm, Y. T. (2015). Antimicrobial peptides of the genus Bacillus: a new era for antibiotics. Can. J. Microbiol. 61, 93-103. doi: 10.1139/cjm-2014-0613

Tambadou, F., Caradec, T., Gagez, A. L., Bonnet, A., Sopena, V., Bridiau, N., et al. (2015). Characterization of the colistin (polymyxin E1 and E2) biosynthetic gene cluster. Arch. Microbiol. 197, 521-532. doi: 10.1007/s00203-015-1084-5

Timmusk, S., Grantcharova, N., and Wagner, E. G. (2005). Paenibacillus polymyxa invades plant roots and forms biofilms. Appl. Environ. Microbiol. 71, 7292-7300. doi: 10.1128/AEM.71.11.7292-7300.2005

Vater, J., Herfort, S., Doellinger, J., Weydmann, M., Lasch, P., and Borriss, R. (2018). Genome mining of lipopeptide biosynthesis of Paenibacillus polymyxa E681 in combination with mass spectrometry - discovery of the lipoheptapeptide paenilipoheptin. ChemBioChem 19, 744-753. doi: 10.1002/cbic.201700615

Winn, M., Fyans, J. K., Zhuo, Y., and Micklefield, J. (2016). Recent advances in engineering nonribosomal peptide assembly lines. Nat. Prod. Rep. 33, 317-347. doi: $10.1039 / \mathrm{c} 5 \mathrm{np} 00099 \mathrm{~h}$

Worner, K., Szurmant, H., Chiang, C., and Hoch, J. A. (2006). Phosphorylation and functional analysis of the sporulation initiation factor Spo0A from Clostridium botulinum. Mol. Microbiol. 59, 1000-1012. doi: 10.1111/j.1365-2958.2005. 04988.x

Xie, N. Z., Li, J. X., Song, L. F., Hou, J. F., Guo, L., Du, Q. S., et al. (2015). Genome sequence of type strain Paenibacillus polymyxa DSM 365, a highly efficient producer of optically active $(R, R)$-2,3-butanediol. J. Biotechnol. 195, 72-73. doi: 10.1016/j.jbiotec.2014.07.441 
Yang, J., Kloepper, J. W., and Ryu, C. M. (2009). Rhizosphere bacteria help plants tolerate abiotic stress. Trends Plant Sci. 14, 1-4. doi: 10.1016/j.tplants.2008.10. 004

Yoon, S. H., Ha, S. M., Kwon, S., Lim, J., Kim, Y., Seo, H., et al. (2017). Introducing EzBioCloud: a taxonomically united database of 16S rRNA gene sequences and whole-genome assemblies. Int. J. Syst. Evol. Microbiol. 67, 1613-1617. doi: $10.1099 /$ ijsem.0.001755

Ziemert, N., Alanjary, M., and Weber, T. (2016). The evolution of genome mining in microbes - a review. Nat. Prod. Rep. 33, 988-1005. doi: 10.1039/c6np $00025 \mathrm{~h}$
Conflict of Interest Statement: The authors declare that the research was conducted in the absence of any commercial or financial relationships that could be construed as a potential conflict of interest.

Copyright (c) 2019 Jeong, Choi, Ryu and Park. This is an open-access article distributed under the terms of the Creative Commons Attribution License (CC BY). The use, distribution or reproduction in other forums is permitted, provided the original author(s) and the copyright owner(s) are credited and that the original publication in this journal is cited, in accordance with accepted academic practice. No use, distribution or reproduction is permitted which does not comply with these terms. 